\title{
RELACIONES DEL REGISTRO TARDÍO DE NACIMIENTOS CON LA CONDICIÓN DE LEGITIMIDAD Y EL TIPO DE UNIÓN DE LOS PADRES*
}

\author{
Beatriz Figueroa
}

El Colegio de México

\section{INTRODUCCIÓN}

UNO DE LOS PROBLEMAS QUE dificulta el conocimiento de los niveles y tendencias de la fecundidad en México es el registro tardío de los nacimientos. El presente trabajo trata un aspecto de este problema, e intenta encontrar una explicación del mismo.

En un estudio anterior, ${ }^{1}$ se consideró como registro tardío cualquier nacimiento inscrito a partir del año de haberse producido; se observó que el porcentaje de registros tardíos aumentaba año con año, con respecto al total de nacimientos registrados y también que este porcentaje era diferente para cada entidad federativa. El periodo estudiado fue de 1950 a 1976.

En el presente trabajo se abarcará el mismo espacio de tiempo y

*Documento preparado para la mesa "Tendencias Recientes en los Patrones Demográficos de América Latina", del IX Congreso Internacional de la Asociación de Estudios Latinoamericanos, septiembre, 1983.

1 B. Figueroa, "El problema del registro tardío de los nacimientos", en Investigación Demográfica en México, 1980, Conscjo Nacional de Ciencia y Tecnologia, 1980. 
se verá si existe alguna relación entre estos hallazgos y la condición de legitimidad, es decir, si el registro tardío de una persona tiene relación con su condición de hijo ilegítimo.

Una primera hipótesis es que una entidad con alto porcentaje de registro de nacimientos considerados como ilegítimos presenta también un alto porcentaje de registro tardío. $Y$ esto se basa en lo siguiente:

a) Suponemos que el número de nacimientos registrados como legítimos dependerá en gran medida del tipo de unión que predomine en la entidad federativa. En una entidad con alto porcentaje de uniones no legales habrá mayor número de nacimientos registrados como ilegítimos que en otra entidad con un porcentaje de uniones libres menor.

b) Suponemos que el tiempo que transcurre para que se registre un nacimiento considerado como ilegítimo es mayor que el que transcurre para registrar a los hijos legítimos. Es de esperar que, en una sociedad en donde la unión de una pareja no necesita estar sancionada por la ley para ser aceptada, se dé poca importancia al registro de los nacimientos de los hijos de estas parejas, ya que éstos son considerados y aceptados por la sociedad de igual manera que sus padres, con toda la legitimidad. Por tanto, lo más probable es que el registro se postergue hasta el momento en que se haga necesaria la presentación de un acta de nacimiento; cuando entre a la escuela primaria, o cuando busque su primer trabajo, lo que causa que el registro del nacimiento sea extemporáneo. Puede suceder también que jamás se lleve a cabo el registro porque nunca le sea requerida el acta de nacimiento. En este tipo de sociedades las uniones libres son tan firmes como las legales; no existen sanciones sociales para estas uniones consensuales, ni para los hijos. Tampoco es necesario tener documentos para identificarse.

c) De acuerdo con algunos estudios sobre la nupcialidad en México se sabe que $42.7 \%$ de las uniones que se inician bajo el régimen de unión consensual, posteriormente se transforman en uniones legales. ${ }^{2}$ Es de suponer que en la fecha en que se legaliza la unión, se registra a los hijos. Por lo tanto el registro es también extemporáneo o tardío, ya que el lapso promedio que transcurre para legalizar una unión que se inició como consensual es de 2.5 años. ${ }^{3}$

2 Julicta Quilodrán, Impacto de la disolución de uniones sobre la fecundidad en México, p. 14 (mimeo).

3 Julieta Quilodrán, "Análisis de la nupcialidad a travès de la historia de 
Los puntos que hemos señalado hasta aquí y que sirvieron para esbozar nuestra hipótesis, también indican los pasos que seguiremos en este trabajo. Este constará de tres partes, en la primera se considerarán las definiciones utilizadas en diferentes épocas, por el registro civil mexicano para la condición de legitimidad de los hijos y la forma como este concepto se asienta en el acta de nacimiento y en las estadísticas sobre el tema.

En la segunda parte del trabajo se presentan los niveles y tendencias de registro de nacimientos legítimos con respecto al total, tanto a nivel nacional como por entidad federativa. En la tercera y última parte se tratará de encontrar una relación a nivel de entidad federativa entre la condición de legitimidad en el registro de nacimientos y el tipo de unión de los padres; posteriormente se estudiará la relación entre la condición de legitimidad y el registro tardío. Esta última relación nos podrá proporcionar una explicación de las tendencias que se observan en el registro tardío al relacionar éste con los diversos tipos de unión que prevalecen en el país.

La forma como intentamos probar nuestra hipótesis tal vez no es la más adecuada, ya que deberíamos observar a las parejas unidas consensualmente, $y$ el comportamiento que siguen para registrar a sus hijos. Tendríamos que observar también si los hijos de estas parejas se registran o no y si al levantar el acta de nacimiento, éstos son declarados como hijos legítimos o ilegítimos y por último tendríamos que saber el tiempo promedio que transcurre entre el nacimiento y su registro. No existe información disponible para hacer un estudio de esa naturaleza ya que sólo se cuenta con estadísticas vitales que están a nivel agregado. Por tanto la información sobre el registro de nacimientos como la condición de legitimidad de las parejas se presentan sólo a nivel de unidades administrativas y nosotros trabajaremos al nivel de entidad federativa.

La información sobre el tipo de unión que utilizaremos proviene de los censos de población, única fuente que proporciona datos sobre los tipos de unión que prevalecen en el país.

uniones", en Investigación Demográfica en México, Consejo Nacional de Ciencia y Tecnología, 1978, pp. 139-149. 


\section{EL REGISTRO DE NACIMIENTOS Y LA CONDICIÓN} DE LEGITIMIDAD SEGÚN EL REGISTRO CIVIL EN MÉXICO ${ }^{4}$

Para el periodo que estamos estudiando hay que tomar en cuenta dos organismos de donde provienen las estadísticas vitales. Por un lado, el registro civil recoge la información primaria de las personas que van a inscribir los hechos vitales, y posteriormente la elaboración de estadísticas se lleva a cabo en la Dirección General de Estadística. La inscripción de los hechos vitales que realiza el registro civil se rige por los ordenamientos contenidos en el Código Civil vigente.

En el caso de México, existe un código que rige para el Distrito y Territorios Federales; a la vez, cada entidad federativa que compone a la república tiene su propio código civil. Esto hace que el registro civil no sea igual en todas las entidades, pero las disposiciones vigentes respecto a los hechos vitales difieren muy poco, de un código a otro, porque casi todos tuvieron como base lo que se consigna en el código federal.

Como el hecho vital que nos interesa en el presente trabajo es el registro de los nacimientos y su condición de legitimidad, a continuación presentaremos un resumen de cómo han evolucionado dichos conceptos a través de las diversas épocas.

1. La primera ley que se dictó sobre el registro civil se denominó, Ley Orgánica del Registro Civil y se remonta a 1857. Esta ley, en su artículo 12 , establecía que se debían registrar nacimientos, matrimonios y defunciones y fue promulgada el 27 de enero del año en cuestión por el presidente Comonfort. No obstante, hay que señalar que ésta no entró en vigor por contravenir el artículo 5o. de la constitución del 5 de febrero, ${ }^{5}$ aunque se menciona en la mayoría de los estudios sobre estos temas, puesto que fue fundamental para la creación del registro civil en México y es el antecedente de las Leyes de Reforma, promulgadas por el presidente Juárez, mismas que en 1873 se incorporaron en calidad de adiciones y reformas a la Constitución de 1857. Sin embargo, la Ley Orgánica del Registro Civil fue adicio-

4 Las siguientes páginas son un breve resumen de lo presentado sobre estos aspectos en el libro que publicó la Secretaría de Gobernación en 1982 , titulado El Registro Civil en México. Antecedentes histórico-legislativo, aspectos juridicos y doctrinarios. Asimismo se consultaron otras publicaciones que aparecen citadas en la bibliografía general.

5 Secretaría de Gobernación, El Registro Civil en México, Antecedentes historico-legislativos, aspectos jurídicos y doctrinarios, Dirección General del Registro Nacional de Población e Identificación Personal, 1982. 
nada por decreto presidencial a la Constitución de 1857 el 25 de septiembre de 1883 y posteriormente se incluyó en la Constitución de $1917 .^{6}$

La Ley Orgánica del Registro Civil del presidente Comonfort contenía cien artículos, agrupados en siete capítulos. E1 capítulo segundo disponía que todo nacimiento ocurrido en territorio nacional debía ser inscrito en el registro civil dentro de las setenta y dos horas siguientes al parto. Terminado ese plazo se impondría una multa a quienes no acataran la disposición; además, el oficial del registro civil ya no podía llevar a cabo la inscripción, a menos que hubiera un mandato judicial.

En cuanto a la legitimidad de los hijos se establecía lo siguiente: en el registro de los críos que nacieran fuera del matrimonio no se podía asentar el nombre del padre, a menos que éste lo solicitara expresamente y siempre y cuando no fuera casado; en este último caso, era imposible la inclusión de su nombre. A los hijos naturales se les registraba anotando el nombre de la madre y los padrinos y se consignaba el nacimiento con la frase "hijo de padre no conocido".

2. La segunda ley sobre el registro civil fue la Ley Orgánica del Registro Civil del presidente Juárez, contenida en las Leyes de Reforma. Consta de cuarenta y tres artículos y un párrafo transitorio, agrupados en cuatro capítulos. El segundo capítulo se refiere a las actas de nacimientos y por primera vez se dispone que se establezcan jueces del estado civil en toda la república.

Esta ley decía que la declaración del nacimiento debía hacerse durante los quince días siguientes al parto, llevando al niño ante el juez del estado civil. En las poblaciones en donde no existiera un juzgado del registro, había que presentarlo a la persona que ejerciera la autoridad local, misma que les daría a los interesados una constancia para llevarla al juez registrador y se levantara el acta correspondiente. En el acta se dejaba constancia de la fecha y lugar de nacimiento, del sexo del recién nacido, del nombre y los datos generales de los padres y de los testigos.

Como se puede observar aquí también se fija un plazo para hacer la declaración de nacimiento, pero no se menciona la condición de legitimidad. Cabe destacar, que en esta ley se especifica que la declaración del nacimiento no sólo es obligación de los padres, sino se señalan también a otras personas como responsables.

6 Rubén Gleason Galicia, Las estadísticas y censos de México, su organización y estado actual, Instituto de Investigaciones Sociales, UNAM, 1968. 
3. Otras leyes que tuvieron mucha influencia en el registro civil mexicano actual fueron las del Código Civil de 1870, que a pesar de haber sido promulgado exclusivamente para el Distrito Federal y el Territorio de Baja California, se extendió en toda la república. Las disposiciones de ese código sustituyeron las de las Leyes de Reforma respecto a las regulaciones sobre el estado civil de las personas y su versión final se encuentra en el código civil de 1884 . Allí se menciona que tanto en el Distrito Federal como en el Territorio de Baja California habría funcionarios que con el cargo de jueces del estado civil, tendrían a su cargo extender las actas relativas al nacimiento, al reconocimiento de hijos y demás actos del estado civil como el matrimonio, la muerte y la tutela de todos los mexicanos y extranjeros residentes en las entidades mencionadas.

En relación con los nacimientos, al igual que en las Leyes de Reforma, se hace necesaria la presentación del recién nacido ante el funcionario del registro civil. También se fija el plazo de quince días para presentarse a levantar el acta para evitar sanciones y se señala que además del padre están obligados a declarar el nacimiento los médicos, cirujanos o matrona que lo hubieran atendido, así como el jefe de la familia de la casa en que se hubiera llevado a cabo el alumbramiento, si éste no se producía en el domicilio de los padres de la criatura. Estas personas tenían un plazo menor que el padre, pues disponían de sólo tres días a partir del nacimiento y en caso de no cumplir serían sujetos a una sanción. Al igual que en las leyes antes citadas, se menciona que en las poblaciones en donde no hubiera juez del estado civil, el niño deberia presentarse ante la persona que ejerciera la autoridad local, quien daría una constancia para que fuera llevada ante el juez correspondiente. En el acta se consignaría el día, hora y lugar de nacimiento, sexo, nombre y apellido del niño. El apellido no se podía omitir y en el caso de hijos de padres desconocidos, el oficjal del registro civil le asignaría uno, lo que constaría en el acta.

Una gran diferencia con las Leyes de Reforma y lo marcado en el código de 1884, se especifica en el capítulo especial sobre los hijos naturales, en donde se señala que si el padre o la madre de un hijo natural, o ambos, le reconocen al presentarlo para su registro dentro del plazo que la ley marca, el acta tendrá el efecto de un reconocimiento legal, pero en ella debe constar que se trata de un hijo natural. De manera que los hijos ilegítimos o adulterinos están identificados en el acta de nacimiento.

4. La siguiente ley que regiría al registro civil surge en 1917. 
Esta legislación se conoce con el nombre de Ley de Relaciones Familiares. Además de regular el vínculo matrimonial, lo hacía con las relaciones de paternidad, filiación, reconocimiento de hijos, patria potestad, emancipación, adopción y tutela. A continuación se resumen los aspectos más relevantes para nuestro estudio de dichas disposiciones. Son considerados hijos legítimos quienes nacen dentro de un matrimonio siempre y cuando el nacimiento ocurra 180 días después de haberse efectuado éste. Como en esta ley se aprobó la disolución del vínculo matrimonial, también se hace referencia a un plazo para considerar como legítimo al hijo de una unión disuelta y este lapso es de no más de 300 días después de la separación. Respecto a los hijos nacidos fuera de matrimonio, son considerados como ilegítimos. La única forma de legitimar según la ley a un hijo nacido fuera del matrimonio, es mediante un subsecuente matrimonio y el reconocimiento del vástago en cualquier momento ya sea en forma explícita o tácita. De manera que es factible decir que, según la ley, los hijos nacidos fuera del matrimonio podrían ser legitimados.

5. Todas estas leyes fueron tomadas en cuenta cuando se formulo el Código Civil de 1928, que es en esencia el que ha servido de base para los códigos actualmente vigentęs en la república. En lo que respecta a los nacimientos, a continuación citaremos los puntos más sobresalientes.

La declaración del nacimiento debe hacerse ante el oficial del registro civil, para lo cual debe presentarse al recién nacido, en la oficina del registro o en el domicilio en donde se hubiera llevado a cabo el nacimiento. En las leyes anteriores la obligación de declarar el nacimiento era del padre o los médicos, o los cirujanos, o la comadrona, o de las personas que hubieran asistido el parto. En este código se menciona por primera vez en forma explícita que la madre también tiene la obligación de declarar el nacimiento de la criatura. Asimismo se alude al plazo, que se amplía a quince días para el padre y a la madre se le dan cuarenta días. También se señalan las sanciones que se aplican cuando no se cumple con este requisito dentro de los plazos establecidos. Al igual que en las pasadas leyes, en caso de no haber un oficial del registro civil en la población que se lleve a cabo el suceso, la declaración del nacimiento debe registrarla la persona que ejerza la autoridad local o municipal y dar una constancia a los interesados para que posteriormente se presente ante el juez respectivo.

En relación con el reconocimiento de los hijos naturales, este código indica que debe estar avalado por el padre y la madre y que el 
término idóneo es de quince días para el padre y cuarenta días para la madre. No obstante, para no perjudicar la situación de los hijos nacidos fuera del matrimonio este reconocimiento puede llevarse a cabo en cualquier fecha. El reconocimiento de los hijos naturales no obstante debe hacerse en el momento de declarar el nacimiento.

Si tomamos en cuenta lo anterior, podemos decir que en teoría todos los nacimientos pueden ser considerados como legítimos sin importar el tipo de unión de los padres siempre y cuando éstos los reconozcan como tal, pero en caso de que no se les reconozca, serár considerados como ilegítimos.

Las principales conclusiones de esta breve revisión de las leyes que han regido al registro civil mexicano son:

a) Desde la primera ley fue motivo de preocupación el plazo en que se debería llevar a cabo la declaración del nacimiento. Los plazos varían desde dos días a partir del nacimiento, hasta cuarenta días.

b) La declaración del nacimiento se debería llevar a cabo ante los of iciales o jueces del registro civil y en caso de no haber una oficina en la población en donde se produjera el hecho, debería ser presentado ante la autoridad máxima de la población.

c) En un principio era sólo obligación del padre presentar la declaración del nacimiento, posteriormente esta obligación se extendió a los médicos o matronas que atendían el parto, así como al jefe de la casa en donde hubiera tenido lugar el evento. El código que rige actualmente hace extensiva la obligación a la madre.

d) Todas aquellas personas que por ley deberían declarar sobre el nacimiento de un niño y que no lo hicieran dentro del plazo señalado por la ley, estarían sujetas a una sanción.

Como podemos ver en los puntos anteriores, existe la obligación de levantar un acta para cada nacimiento y además se fija un plazo para que se realice esta tarea, de lo contrario se fijan sanciones. No obstante, gran porcentaje de la población mexicana no cumple con este requerimiento, dando lugar a numerosos registros tardíos.

En cuanto al concepto de legitimidad llegamos a la siguiente conclusión:

En las primeras leyes todo hijo nacido fuera del matrimonio era considerado ilegítimo aunque el padre lo quisiera reconocer como descendiente de él. Posteriormente esto se fue modificando hasta que en las leyes actuales todo hijo puede ser registrado como legítimo independientemente del estado civil de los padres, siempre y cuando éstos lo reconozcan como tal al momento de registrar su nacimiento. 
Esta última conclusión es de suma importancia para nuestro estudio, pues pese a que existe la posibilidad de que desde 1928 no haya nacimientos considerados como ilegítimos todavía en 1975 podemos observar que muchos de los nacimientos registrados son considerados como tal.

\section{NIVELES Y TENDENCIAS DE LOS REGISTROS DE NACIMIENTOS SEGÚN SU CONDICIÓN DE LEGITIMIDAD}

La Dirección General de Estadística, organismo responsable de elaborar y difundir las estadísticas de los hechos vitales, publicó a partir del año de 1952, año con año y hasta 1975, una tabulación sobre el registro de nacimientos según su calidad de legitimidad.

Según la nota que aparece al pie de las tabulaciones, en el procesamiento de la información y para la elaboración de la misma se considera como hijo legítimo de los años 1962 a 1971, a los hijos de padres casados civilmente. De 1972 a 1975 , además de los señalados en el renglón anterior, se incluyeron como legítimos a los hijos provenientes de padres casados por la Iglesia. De 1952 a 1961 no se indicó en las tabulaciones el concepto que se utilizó para distinguir la condición de legitimidad.

Al examinar las formas que la Dirección General de Estadística envía a las oficialías del registro civil, así como las instrucciones para llenarlas pudimos observar que en los formularios correspondientes a los nacimientos no existe ningún renglón sobre la condición de legitimidad. Este espacio debería encontrarse para que quedara asentada la condición de legitimidad que aparece en el acta de nacimiento, de acuerdo con lo prescrito en los códigos civiles vigentes. En su lugar, en esta hoja y en las instrucciones, sólo se indica que se inscriba el tipo de unión de los padres (solteros, casados por lo civil, casados por la Iglesia, viudos, divorciados, unión libre y se ignora), de ahí que para la tabulación sea necesario recurrir a este rubro e identificar la condición de legitimidad del infante registrado. ${ }^{7}$

7 Dirección General de Estadística, Programas y procedimientos, serie II, núms. 2 y 10.

a) "Normas para las publicaciones del área de estad isticas demográficas y sociales", 1975, p. 10

b) "Instrucciones para llenar en el Registro Civil los formularios estađísticos de los hechos vitales", 1975, p. 8. 
Señalamos esta contradicción entre lo establecido por la ley y la forma de conocer el estado de legitimidad de los hijos, ya que puede ser muy útil para analizar los niveles y las tendencias sobre la condición de legitimidad en el registro de nacimientos.

En el cuadro 1 se presentan las proporciones para el total del país del registro de nacimientos considerados como ilegítimos, según la conceptualización de la Dirección General de Estadística. Estos datos muestran que no ha habido grandes cambios en la proporción de nacimientos legítimos a nivel nacional, pues de 1951 a 1973 se ha mantenido alrededor de 75 u $80 \%$. Las pequeñas fluctuaciones que se dan de un año a otro se pueden observar mejor en la gráfica 1 , en la que también puede verse que no hay una tendencia clara de aumento o descenso de esta proporción.

Los años 1974 y 1975 no se consideraron junto con los mencionados ya que no son estrictamente comparables. En estos dos años no se incluyeron los nacimientos registrados en forma extemporánea. En cambio, de 1951 a 1973 se utilizaron todos los nacimientos registrados, sin distinguir si habían ocurrido en el año del registro o en años anteriores. Es importante destacar lo que sucede en estos dos años respecto al periodo anterior, puesto que hay un aumento de $12 \%$ en la proporción de nacimientos registrados como legítimos.

La pregunta inmediata que surge es, ¿cuál será la causa de este aumento? Ya en párrafos anteriores habíamos señalado que en la forma de captación de la información sobre la legitimidad del registrado, se habían producido algunos cambios. No obstante, para los últimos 4 años se utilizó la misma definición y el aumento fue sólo relevante para los últimos 2; de manera que la explicación es la exclusión del registro tardío que se hizo para 1974 y 1975 . Este hallazgo puede ser un punto a favor de nuestra hipótesis, pero de ninguna manera esuna comprobación de la misma.

Como deseábamos saber si a nivel de las entidades el porcentaje de nacimientos legítimos era similar al del total del país, analizamos los datos correspondientes a las entidades federativas. En el cuadro 2 presentamos, para los años 1951, 1955, 1960, 1965 y 1970, el porcentaje de nacimientos registrados como legítimos e incluimos los datos para 1975, aunque como hemos señalado no son estrictamente comparables. Para analizar la información del cuadro 2 utilizamos el "análisis exploratorio de datos", que privilegia a la mediana como medida de tendencia central y define además sus propios indicadores para reconocer la dispersión de los datos.

El resultado del "análisis exploratorio" se presenta en el cuadro 
Cuadro 1. México: Distribución porcentual de los nacimientos registrados según condición de legitimidad: 1951-1975

\begin{tabular}{lcccc}
\hline Año & $\begin{array}{c}\text { Total de nacimientos } \\
\text { registrados }\end{array}$ & $\begin{array}{c}\text { Total nacimientos } \\
\text { registrados como } \\
\text { ilegítimos }\end{array}$ & $\begin{array}{c}\text { Distribución } \\
\text { porcentual } \\
\text { ilegítimos }\end{array}$ & $\begin{array}{c}\text { Distribución } \\
\text { porcentual } \\
\text { legitimos }\end{array}$ \\
\hline 1951 & 1183788 & 322857 & 27.27 & 72.73 \\
1952 & 1261775 & - & - & - \\
1953 & 1339837 & 352192 & 27.91 & 72.09 \\
1954 & 1377917 & 332548 & 24.82 & 75.18 \\
1955 & 1427722 & 320509 & 23.26 & 77.74 \\
1956 & 1485202 & 321742 & 22.54 & 76.45 \\
1957 & 1447578 & 349798 & 23.55 & 75.60 \\
1958 & 1589606 & 353206 & 24.40 & 74.59 \\
1959 & 1608174 & 403971 & 25.41 & 75.32 \\
1960 & 1647006 & 402618 & 25.04 & 74.95 \\
1961 & 1705481 & 406561 & 25.05 & 75.15 \\
1962 & 1756624 & 427283 & 24.85 & 75.43 \\
1963 & 1849408 & 436608 & 24.57 & 76.82 \\
1964 & 1888171 & 454342 & 24.35 & 76.57 \\
1965 & 1954340 & 459780 & 23.18 & 72.55 \\
1966 & 1981363 & 452991 & 23.43 &
\end{tabular}




\begin{tabular}{lllll}
1969 & 2088902 & 478595 & 22.91 & 77.09 \\
1970 & 2132630 & 583222 & 27.35 & 72.65 \\
1971 & 2231399 & 503140 & 22.55 & 77.45 \\
1972 & 2346002 & 434431 & 18.52 & 81.48 \\
1973 & 2572287 & 522935 & 20.33 & 79.67 \\
1974 & $1635010^{2}$ & $120856^{3}$ & 7.39 & 92.61 \\
1975 & $1621124^{2}$ & $120220^{3}$ & 7.42 & 92.58 \\
\hline
\end{tabular}

Fuente: Anuarios Estadisticos de los Estados Unidos Mexicanos, 1951-1971, Dirección General de Estadística.

1 No hay información publicada para 1952.

2 Total de nacimientos registrados y ocurridos ese mismo año. Esta información es diferente a la que aparece en años anteriores en los que se incluía el total de nacimientos registrados.

3 Total de nacimientos registrados como ilegítimos (nacimientos que ocurrieron el mismo año que el del registro). Esta cifra es -enor que en años anteriores (véase nota 2). 
GRÁFICA 1

Distribución porcentual de los nacimientos registrados como ilegitimos del total
de nacimientos registrados. Total del Puss 1951, 1953-1975.

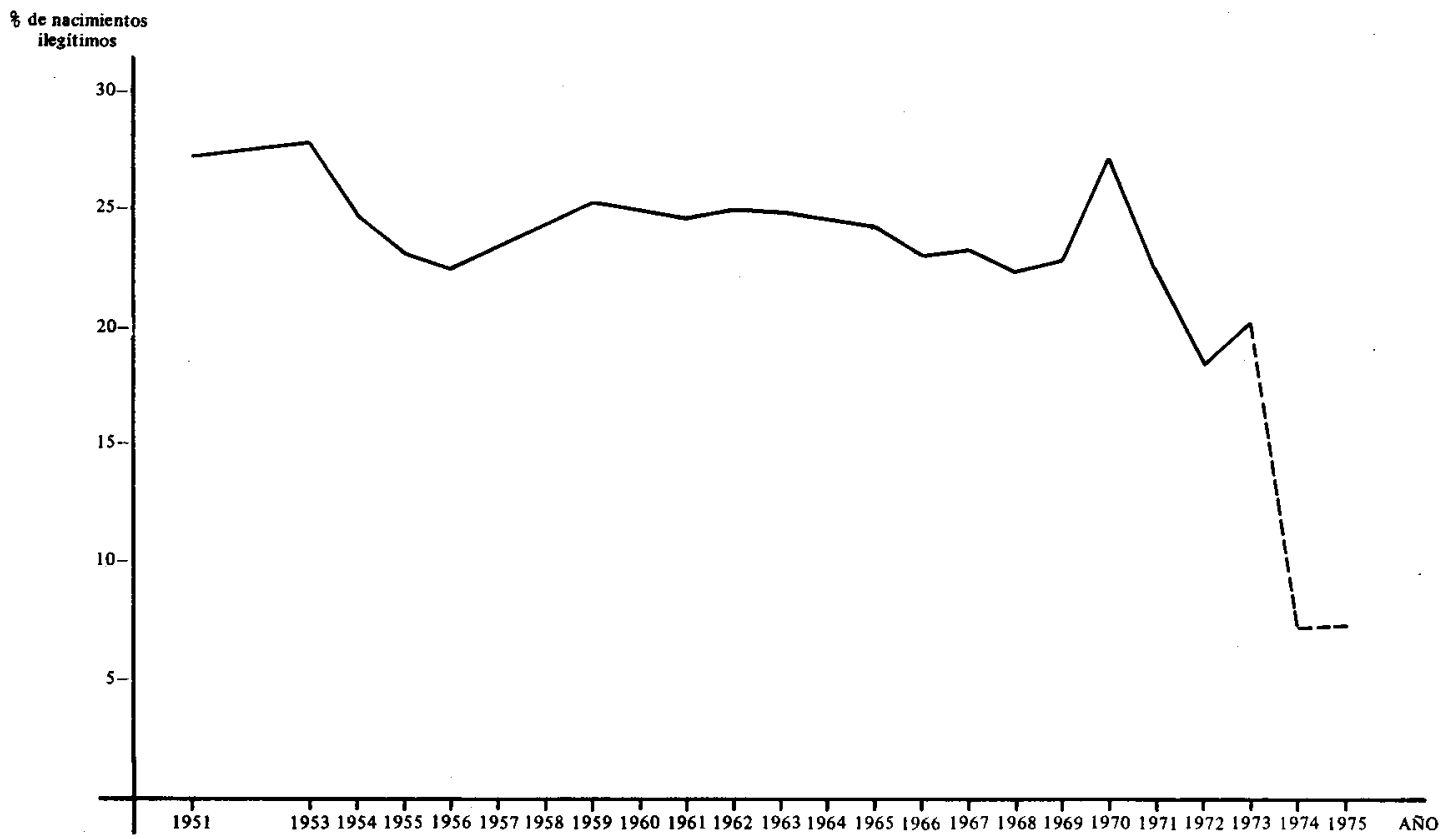


GRAFICA 2 Distribucion porcentual de los nacimientos reasietrados como befitimos del total

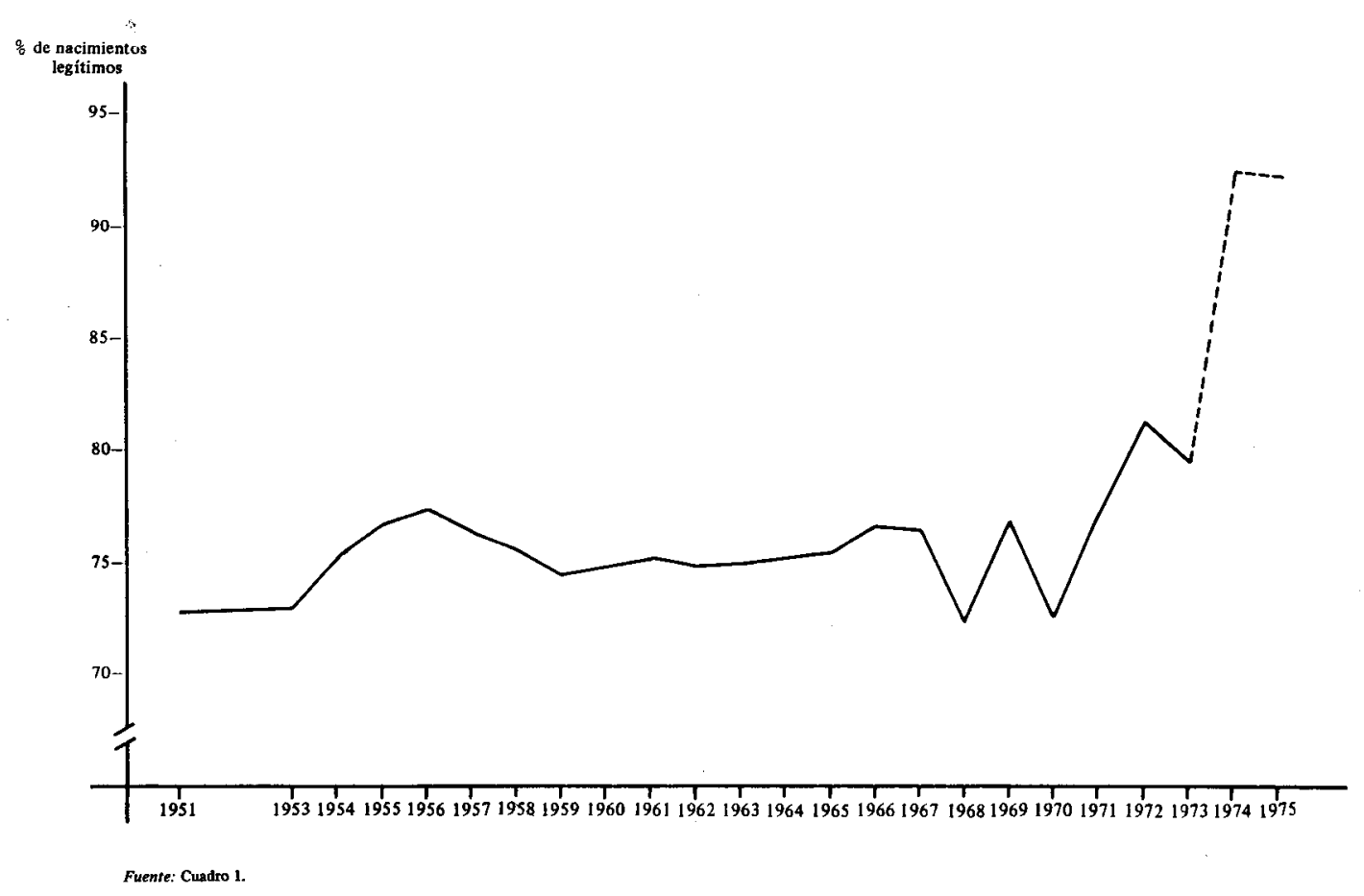


Cuadro 2. México: Distribución porcentual de los nacimientos registrados como legítimos por entidad federativa: $1951,1955,1960,1965,1970$ y 1975.1

\begin{tabular}{lllllll}
\hline Entidad Federativa & 1951 & 1955 & 1960 & 1965 & 1970 & $1975^{2}$ \\
\hline Total del país & 72.73 & 76.74 & 74.96 & 75.65 & 72.65 & 92.58 \\
1. Aguascalientes & 91.45 & 94.17 & 90.59 & 91.08 & 88.79 & 97.14 \\
2. Baja California Norte & 78.14 & 78.38 & 79.49 & 82.23 & 85.93 & 91.37 \\
3. Baja California Sur & 61.01 & 66.41 & 67.60 & 70.45 & 76.41 & 94.96 \\
4. Campeche & 78.67 & 76.61 & 78.32 & 78.85 & 78.32 & 95.39 \\
5. Coahuila & 89.07 & 89.38 & 91.16 & 91.49 & 85.64 & 97.87 \\
6. Colima & 80.92 & 73.07 & 72.13 & 71.28 & 75.21 & 90.82 \\
7. Chiapas & 39.66 & 54.33 & 47.36 & 51.48 & 54.81 & 89.08 \\
8. Chihuahua & 86.24 & 83.80 & 84.75 & 83.77 & 88.77 & 89.93 \\
9. Distrito Federal & 83.10 & 85.47 & 85.79 & 87.47 & 66.26 & 94.14 \\
10. Durango & 76.37 & 80.59 & 80.95 & 80.43 & 77.42 & 96.21 \\
11. Guanajuato & 82.75 & 83.23 & 84.31 & 83.13 & 86.57 & 96.38 \\
12. Guerrero & 76.64 & 79.32 & 80.85 & 82.53 & 76.59 & 97.88 \\
13. Hidalgo & 42.77 & 56.83 & 49.16 & 52.34 & 57.78 & 89.72 \\
14. Jalisco & 88.47 & 89.49 & 90.30 & 90.55 & 83.00 & 95.78 \\
15. México & 72.76 & 75.06 & 74.67 & 74.98 & 70.76 & 94.50 \\
16. Michoacán & 87.28 & 88.12 & 87.24 & 85.87 & 86.11 & 97.28 \\
17. Morelos & 62.46 & 63.76 & 63.87 & 67.57 & 70.24 & 90.80 \\
18. Nayarit & 51.47 & 63.09 & 54.87 & 59.78 & 59.24 & 80.55 \\
19. Nuevo León & 93.94 & 92.39 & 94.46 & 93.95 & 93.16 & 97.27 \\
20. Oaxaca & 50.74 & 62.78 & 49.64 & 48.68 & 52.19 & 78.52 \\
21. Puebla & 62.10 & 68.80 & 63.34 & 63.94 & 60.94 & 91.40 \\
22. Querétaro & 92.42 & 91.14 & 91.13 & 89.41 & 90.13 & 95.82
\end{tabular}




\begin{tabular}{lllllll} 
23. Quintana Roo & 64.28 & 75.41 & 72.42 & 78.96 & 84.45 & 95.51 \\
24. San Luis Potosi & 79.90 & 81.45 & 84.01 & 83.59 & 81.55 & 95.02 \\
25. Sinaloa & 49.53 & 52.13 & 53.34 & 57.10 & 62.65 & 90.02 \\
26. Sonora & 73.42 & 72.72 & 73.59 & 75.57 & 75.08 & 88.58 \\
27. Tabasco & 47.17 & 54.93 & 54.97 & 61.21 & 62.29 & 92.35 \\
28. Tamaulipas & 81.91 & 79.17 & 76.28 & 73.82 & 73.09 & 97.34 \\
29. Tlaxcala & 75.63 & 77.94 & 78.48 & 80.15 & 74.75 & 98.61 \\
30. Veracruz & 40.20 & 57.20 & 44.04 & 49.01 & 51.12 & 77.72 \\
31. Yucatán & 73.13 & 75.86 & 77.01 & 79.25 & 78.97 & 94.03 \\
32. Zacatecas & 90.79 & 91.36 & 91.78 & 92.22 & 92.88 & 99.07 \\
\hline
\end{tabular}

Fuente: Anuarios Estadisticos de los Estados Unidos Mexicanos, 1951-1975, Dirección General de Estadística.

1 Los porcentajes de nacimientos legítimos se obtuvieron dividiendo el total de nacimientos registrados como legítimos entre el total de nacimientos registrados.

2 Para 1975 el porcentaje se obtuvo dividiendo el total de nacimientos registrados como legítimos, que ocurrieron ese año, entre el total de nacimientos ocurridos y registrados. 
3. En éste podemos observar que la mediana de la proporción de registro de nacimientos legítimos está entre 75 y 80 para el periodo considerado, es decir no hay gran variación de un quinquenio a otro, lo que indica que no ha habido cambios importantes en esta proporción. Las diferencias entre las entidades se mantienen o al menos eso parecen indicar los valores de las bisagras, que es el valor medio que se encuentra entre los extremos y la mediana, ya que tanto en el valor inferior como en el superior los porcentajes son similares para todos los años considerados. Los valores observados son entre 61 y 64 para las bisagras inferiores y de 83 a 86 para las bisagras superiores. En cuanto a la dispersión de los porcentajes para cada entidad, suponemos que tampoco ha habidograndes cambios, ya que el rango, que es la diferencia entre valores extremos, es semejante en los cinco periodos y a lo más se observan fluctuaciones pero no una tendencia definida; 54.2 en 1950, para descender a 42.1 en 1955 y al quinquenio siguiente volver a subir a 54.1 y volver a bajar cinco años después a 45.3 y mantenerse en los 40 para 1970. Debido a estas fluctuaciones no se puede decir que haya una tendencia clara de aumento o de disminución de las diferencias entre entidades.

El año 1975 lo consideramos en forma separada, por no poderse comparar con los años anteriores. Para este año vemos que la mediana de la proporción de nacimientos legítimos de 94.8 , está por encima de años anteriores. También que la dispersión de los datos es menor, el valor de las bisagras es muy cercano al de la mediana y el rango es casi la mitad del valor que para los años anteriores.

Las conclusiones que podemos obtener de ese cuadro son las siguientes:

a) El nivel promedio de registro de nacimientos legítimos de 1951 a 1970 está entre 75 y $80 \%$.

b) Al suprimir el registro tardío para obtener el porcentaje de los nacimientos legítimos el promedio se eleva a casi $95 \%$, es decir entre 10 y $15 \%$ más que los años anteriores.

c) Las diferencias entre las entidades se mantienen para el periodo 1951 a 1970, ya que el valor de las bisagras y del rango son muy similares para las cinco series de datos.

d) Al suprimir el registro tardfo, para obtener la proporción de nacimientos legítimos, las diferencias entre las entidades disminuyen. Tanto los valores de las bisagras como el del rango así lo indican, especialmente el rango se disminuye a casi la mitad del valor que para los años anteriores.

A partir de las conclusiones anteriores, consideramos convenien- 
Cuadro 3. Resumen de las medidas obtenidas en el análisis exploratorio de los porcentajes de los nacimientos registrados como legítimos: 1951-1975

\begin{tabular}{|c|c|c|c|c|c|c|c|}
\hline \multirow{3}{*}{ Año } & \multirow{3}{*}{$\begin{array}{l}\text { Número de } \\
\text { observaciones }\end{array}$} & \multirow{3}{*}{ Mediana } & \multicolumn{4}{|c|}{ Medidas del análisis exploratorio } & \multirow{3}{*}{ Rango } \\
\hline & & & \multicolumn{2}{|c|}{ Bisagras $^{2}$} & \multicolumn{2}{|c|}{ Valores extremos } & \\
\hline & & & Inferior & Superior & Inferior & Superior & \\
\hline 1951 & 32 & 76.5 & 61.0 & 86.2 & 39.7 & 93.9 & 54.2 \\
\hline 1955 & 32 & 77.3 & 63.8 & 83.8 & 52.1 & 94.2 & 42.1 \\
\hline 1960 & 32 & 77.7 & 63.3 & 85.8 & 40.4 & 94.5 & 54.1 \\
\hline 1965 & 32 & 79.0 & 63.9 & 85.9 & 48.7 & 94.0 & 45.3 \\
\hline 1970 & 32 & 76.5 & 62.7 & 85.9 & 51.1 & 93.2 & 42.1 \\
\hline 1975 & 32 & 94.8 & 90.0 & 97.1 & 77.7 & 99.1 & 7.1 \\
\hline
\end{tabular}

Fuente: Cálculos elaborados con base en los datos del cuadro 2.

1 En el cálculo se tomaron las 32 entidades federativas.

2 Se denomina bisagra al valor medio que se encuentra entre los valores extremos y la mediana. 
te estudiar cada entidad por separado. Para ello utilizamos la misma metodología del "análisis exploratorio de datos" y analizamos la información entidad por entidad, para los periodos 1951-1973 y 1951-1975. El propósito de este análisis era encontrar grupos de entidades que tuvieran un comportamiento similar respecto al nivel y a las tendencias sobre la proporción de registro de nacimientos legítimos.

El resultado del análisis para el periodo $1951-1973$ se presenta en el cuadro 4, y para $1951-1975$ en el cuadro 5. Como podemos observar, ambas series clasifican a los estados en seis grupos y cada grupo queda conformado por las mismas entidades federativas. A continuación describimos las características de cada uno de los grupos.

\section{Grupo I}

El grupo I está compuesto por seis entidades federativas, dos estados del norte (Nuevo León y Coahuila) y cuatro del centro (Jalisco, Querétaro, Aguascalientes y Zacatecas). Este grupo tiene una mediana de más de 90 y la dispersión en la proporción es muy pequeña. Es decir, que en el periodo considerado la proporción de nacimientos legítimos es casi constante y con pocas variaciones, según lo muestran los valores de las bisagras, las distancias (M) y el rango. Estas entidades son las que tienen la proporción más alta de nacimientos legítimos y para poder interpretar este hecho adecuadamente habría que profundizar en un análisis socioeconómico de estas entidades, lo que rebasa los objetivos de este trabajo, pero consideramos indispensable para explicar la agrupación que obtenemos. Lo único que podemos decir es que estos estados son relativamente similares en algunas características económicas.

\section{Grupo II}

Este grupo está compuesto por ocho entidades, cuatro del norte (Baja California Norte, Durango, San Luis Potosí y Chihuahua), tres del centro (Guanajuato, Michoacán y el Distrito Federal) y una del sur (Guerrero). La mediana es del orden de 80 a 87 , no llega a 90 y también la dispersión es pequeña. Por lo tanto, se puede decir que estas entidades tienen una proporción de nacimientos legítimos un 
Cuadro 4. México: Medidas obtenidas en el análisis exploratorio de datos (mediana, bisagras, distancia entre mediana y bisagras (M), valores extremos y rango) por grupos de entidades: 1951-1973

(Series de porcentajes en 22 años) 1

Grupo I

\begin{tabular}{|c|c|c|c|c|c|c|c|c|}
\hline \multirow{2}{*}{$\begin{array}{l}\text { Entidad } \\
\text { Federativa }\end{array}$} & \multirow[t]{2}{*}{ Mediana } & \multicolumn{2}{|c|}{ Bisagras $^{2}$} & \multicolumn{2}{|c|}{ Distancia (M) } & \multicolumn{2}{|c|}{ Valores extremos } & \multirow[t]{2}{*}{ Rango } \\
\hline & & Inferior & Superior & Inferior & Superior & Inferior & Superior & \\
\hline 1. Jalisco & 90.1 & 88.9 & 91.1 & 1.2 & 1.0 & 83.0 & 93.3 & 10.3 \\
\hline 2. Querétaro & 90.7 & 89.6 & 91.4 & 1.1 & 0.7 & 84.6 & 93.9 & 9.3 \\
\hline 3. Coahuila & 91.2 & 89.4 & 91.8 & 1.8 & 0.6 & 85.6 & 95.9 & 10.3 \\
\hline 4. Zacatecas & 92.1 & 91.7 & 92.4 & 0.4 & 0.3 & 85.0 & 94.7 & 97 \\
\hline 5. Aguascalientes & 92.3 & 91.1 & 93.9 & 1.2 & 1.6 & 85.4 & 94.6 & 9.2 \\
\hline 6. Nuevo León & 94.0 & 93.6 & 94.6 & 0.4 & 0.9 & 87.2 & 96.6 & 9.4 \\
\hline \multicolumn{9}{|c|}{ Grupo II } \\
\hline Entidad & Mediana & \multicolumn{2}{|c|}{ Bisagras $^{2}$} & \multicolumn{2}{|c|}{ Distancia (M) } & \multicolumn{2}{|c|}{ Valores extremos } & Rango \\
\hline Federativa & & Inferior & Superior & Inferior & Superior & Inferior & Superior & \\
\hline 1. Baja Calif. Nte. & 80.3 & 78.1 & 83.3 & 2.2 & 3.0 & 75.8 & 86.2 & 10.4 \\
\hline 2. Durango & 80.7 & 80.2 & 82.2 & 0.5 & 1.5 & 76.4 & 87.2 & 10.8 \\
\hline 3. Guerrero & 81.0 & 79.4 & 83.9 & 1.6 & 2.9 & 76.6 & 87.7 & 11.1 \\
\hline 4. San Luis & & & & & & & & \\
\hline Potosí & 83.6 & 81.6 & 84.1 & 2.0 & 0.5 & 79.4 & 86.6 & 7.2 \\
\hline
\end{tabular}


Cuadro 4. México: Medidas obtenidas en el análisis exploratorio de datos (mediana, bisagras, distancia entre mediana y bisagras (M), valores extremos y rango) por grupo de entidades: 1951-1973

(Series de porcentajes en 22 años) ${ }^{1}$ (continuación)

\begin{tabular}{|c|c|c|c|c|c|c|c|c|}
\hline \multirow{2}{*}{$\begin{array}{l}\text { Entidad } \\
\text { Federativa }\end{array}$} & \multirow{2}{*}{ Mediana } & \multicolumn{2}{|c|}{ Bisagras $^{2}$} & \multicolumn{2}{|c|}{ Distancia (M) } & \multicolumn{2}{|c|}{ Valores extremos } & \multirow[t]{2}{*}{ Rango } \\
\hline & & Inferior & Superior & Inferior & Superior & Inferior & Superior & \\
\hline 5. Guanajuato & 84.1 & 82.8 & 86.6 & 1.3 & 2.5 & 81.9 & 91.5 & 9.6 \\
\hline 6. Chihuahua & 85.6 & 84.4 & 86.2 & 1.2 & 0.6 & 82.8 & 89.9 & 7.1 \\
\hline 7. Distrito & & & & & & & & \\
\hline Federal & 85.7 & 84.7 & 87.7 & 1.0 & 2.0 & 66.3 & 91.3 & 25.0 \\
\hline 8. Michoacán & 86.8 & 85.9 & 87.3 & 0.9 & 0.5 & 77.4 & 89.0 & 11.6 \\
\hline \multicolumn{9}{|c|}{ Grupo III } \\
\hline Entidad & Mediana & \multicolumn{2}{|c|}{ Bisagras $^{2}$} & \multicolumn{2}{|c|}{ Distancia (M) } & \multicolumn{2}{|c|}{ Valores extremos } & Rango \\
\hline Federativa & & Inferior & Superior & Inferior & Superior & Inferior & Superior & \\
\hline 1. Colima & 72.4 & 71.3 & 73.9 & 1.1 & 1.5 & 64.9 & 80.9 & 16.0 \\
\hline 2. Sonora & 74.7 & 73.4 & 76.0 & 1.3 & 1.3 & 71.7 & 84.9 & 13.2 \\
\hline 3. Tamaulipas & 75.9 & 74.0 & 77.8 & 1.9 & 1.9 & 70.4 & 81.9 & 11.5 \\
\hline 4. Quintana Roo & 75.6 & 72.7 & 80.3 & 2.9 & 4.7 & 64.7 & 88.8 & 24.1 \\
\hline 5. México & 77.3 & 75.0 & 80.7 & 2.3 & 3.4 & 70.8 & 84.9 & 14.1 \\
\hline 6. Campeche & 78.3 & 77.9 & 79.7 & 0.4 & 1.4 & 73.4 & 84.2 & 108 \\
\hline 7. Tlaxcala & 78.8 & 77.9 & 80.2 & 0.9 & 1.4 & 74.8 & 86.0 & 11.2 \\
\hline 8. Yucatán & 78.4 & 77.0 & 81.0 & 1.4 & 2.6 & 73.1 & 86.6 & 13.5 \\
\hline
\end{tabular}


Cuadro 4. México: Medidas obtenidas en el análisis exploratorio de datos (mediana, bisagras, distancia entre mediana y bisagras (M), valores extremos y rango) por grupo de entidades: 1951.1973

(Serie de porcentajes en 22 años) ${ }^{1}$ (continuación)

Grupo IV

\begin{tabular}{|c|c|c|c|c|c|c|c|c|}
\hline \multirow{2}{*}{$\begin{array}{l}\text { Entidad } \\
\text { Federativa }\end{array}$} & \multirow[t]{2}{*}{ Mediana } & \multicolumn{2}{|c|}{ Bisagras $^{2}$} & \multicolumn{2}{|c|}{ Distancia (M) } & \multicolumn{2}{|c|}{ Valores extremos } & \multirow[t]{2}{*}{ Rango } \\
\hline & & Inferior & Superior & Inferior & Superior & Inferior & Superior & \\
\hline 1. Morelos & 65.1 & 63.4 & 67.6 & 1.7 & 2.5 & 59.7 & 75.9 & 16.2 \\
\hline 2. Puebla & 66.0 & 63.3 & 67.6 & 2.7 & 1.6 & 59.3 & 77.6 & 18.3 \\
\hline 3. Baja Calif. Sur & 68.7 & 67.6 & 74.9 & 1.1 & 6.2 & 61.0 & 82.1 & 21.1 \\
\hline \multicolumn{9}{|c|}{ Grupo V } \\
\hline \multirow{2}{*}{$\begin{array}{l}\text { Entidad } \\
\text { Federativa }\end{array}$} & \multirow[t]{2}{*}{ Mediana } & \multicolumn{2}{|c|}{ Bisagras $^{2}$} & \multicolumn{2}{|c|}{ Distancia (M) } & \multicolumn{2}{|c|}{ Valores extremos } & Rango \\
\hline & & Inferior & Superior & Inferior & Superior & Inferior & Superior & \\
\hline 1. Hidalgo & 52.2 & 50.3 & 56.8 & 1.9 & 4.6 & 42.8 & 62.2 & 19.4 \\
\hline 2. Chiapas & 52.4 & 49.0 & 54.9 & 3.4 & 2.5 & 39.7 & 61.2 & 21.5 \\
\hline 3. Sinaloa & 55.4 & 52.1 & 61.5 & 3.3 & 6.1 & 49.5 & 67.1 & 17.6 \\
\hline 4. Nayarit & 58.2 & 55.8 & 59.8 & 2.4 & 1.6 & 51.5 & 67.1 & 15.6 \\
\hline 5. Tabasco & 57.9 & 55.5 & 62.3 & 2.4 & 4.4 & 47.2 & 72.0 & 24.8 \\
\hline
\end{tabular}


Cuadro 4. México: Medidas obtenidas en el análisis exploratorio de datos (mediana, bisagras, distancia entre mediana y bisagras (M), valores extremos y rango) por grupo de entidades: 1951-1973

(Series de porcentajes en 22 años) ${ }^{1}$ (conclusión)

Grupo VI

\begin{tabular}{lllllllll}
\hline \multirow{2}{*}{$\begin{array}{l}\text { Entidad } \\
\text { Federativa }\end{array}$} & Mediana & \multicolumn{2}{c}{ Bisagras $^{2}$} & \multicolumn{2}{c}{ Distancia (M) } & \multicolumn{2}{c}{ Valores extremos } & Rango \\
& & Inferior & Superior & Inferior & Superior & Inferior & Superior & \\
\hline 1. Veracruz & 49.2 & 45.0 & 53.2 & 4.2 & 4.0 & 40.2 & 60.8 & 20.6 \\
2. Oaxaca & 49.3 & 47.7 & 53.4 & 1.6 & 4.1 & 45.8 & 64.6 & 18.8 \\
\hline
\end{tabular}

Fuente: Cálculos elaborados con base en datos de los Anuarios Estadisticos de los Estados Unidos Mexicanos, 1951-1973, Dirección

General de Estad ística.
1 En el cálculo de las medidas se tomaron en cuenta series de pocentajes de 22 años: los años 1951 y los del periodo 1953-1973.

2 Véase nota 2 del cuadro 3. 
Cuadro 5. México: Medidas obtenidas en el análisis exploratorio de datos (mediana, bisagras, distancia entre mediana y bisagras $(M)$, valores extremos y rango) por grupos de entidades federativas, 1951-1975

(Series de porcentajes en 24 años) ${ }^{1}$

Grupo I

\begin{tabular}{|c|c|c|c|c|c|c|c|c|}
\hline \multirow{2}{*}{$\begin{array}{l}\text { Entidad } \\
\text { Federativa }\end{array}$} & \multirow[t]{2}{*}{ Mediana } & \multicolumn{2}{|c|}{ Bisagras $^{2}$} & \multicolumn{2}{|c|}{ Distancia (M) } & \multicolumn{2}{|c|}{ Valores extremos } & \multirow[t]{2}{*}{ Rango } \\
\hline & & Inferior & Superior & Inferior & Superior & Inferior & Superior & \\
\hline 1. Jalisco & 90.1 & 88.9 & 91.5 & 1.3 & 1.3 & 83.0 & 96.7 & 13.7 \\
\hline 2. Querétaro & 90.8 & 89.6 & 92.3 & 1.2 & 1.5 & 84.6 & 95.8 & 11.2 \\
\hline 3. Coahuila & 91.3 & 89.4 & 92.2 & 1.9 & 0.9 & 85.6 & 97.9 & 12.3 \\
\hline 4. Zacatecas & 92.2 & 91.7 & 93.2 & 0.5 & 1.0 & 85.0 & 99.1 & 14.1 \\
\hline 5. Aguascalientes & 92.9 & 91.1 & 94.2 & 1.8 & 1.3 & 85.4 & 97.2 & 11.8 \\
\hline 6. Nuevo León & 94.1 & 93.6 & 95.4 & 0.5 & 1.3 & 87.2 & 97.2 & 10.0 \\
\hline
\end{tabular}

Grupo II

\begin{tabular}{|c|c|c|c|c|c|c|c|c|}
\hline \multirow{2}{*}{$\begin{array}{l}\text { Entidad } \\
\text { Federativa }\end{array}$} & \multirow[t]{2}{*}{ Mediana } & \multicolumn{2}{|c|}{ Bisagras $^{2}$} & \multicolumn{2}{|c|}{ Distancia (M) } & \multicolumn{2}{|c|}{ Valores extremos } & \multirow[t]{2}{*}{ Rango } \\
\hline & & Inferior & Superior & Inferior & Superior & Inferior & Superior & \\
\hline $\begin{array}{l}\text { 1. Baja Calif. Nte. } \\
\text { 2. Durango } \\
\text { 3. Guerrero } \\
\text { 4. San Luis Potosí }\end{array}$ & $\begin{array}{l}80.8 \\
80.9 \\
81.2 \\
83.6\end{array}$ & $\begin{array}{l}78.1 \\
80.2 \\
79.4 \\
81.6\end{array}$ & $\begin{array}{l}83.9 \\
83.8 \\
85.3 \\
84.2\end{array}$ & $\begin{array}{l}2.7 \\
0.7 \\
1.8 \\
2.0\end{array}$ & $\begin{array}{l}3.1 \\
2.9 \\
4.1 \\
0.6\end{array}$ & $\begin{array}{l}75.8 \\
76.4 \\
76.6 \\
79.4\end{array}$ & $\begin{array}{l}92.4 \\
96.2 \\
98.4 \\
95.0\end{array}$ & $\begin{array}{l}16.6 \\
19.8 \\
21.8 \\
15.6\end{array}$ \\
\hline
\end{tabular}


Cuadro 5. México: Medidas obtenidas en el análisis exploratorio de datos (mediana, bisagras, distancia, entre mediana y bisagras (M), valores extremos y rango) por grupos de entidades federativas, 1951-1975

(Series de porcentajes en 24 años) ${ }^{1}$ (continuación)

\begin{tabular}{|c|c|c|c|c|c|c|c|c|}
\hline \multirow{2}{*}{$\begin{array}{l}\text { Entidad } \\
\text { Federativa }\end{array}$} & \multirow[t]{2}{*}{ Mediana } & \multicolumn{2}{|c|}{ Bisagras $^{2}$} & \multicolumn{2}{|c|}{ Distancia (M) } & \multicolumn{2}{|c|}{ Valores extremos } & \multirow[t]{2}{*}{ Rango } \\
\hline & & Inferior & Superior & Inferior & Superior & Inferior & Superior & \\
\hline $\begin{array}{l}\text { 5. Guanajuato } \\
\text { 6. Chihuahua } \\
\text { 7. Distrito }\end{array}$ & $\begin{array}{l}84.3 \\
85.8\end{array}$ & $\begin{array}{l}82.8 \\
84.4\end{array}$ & $\begin{array}{l}87.4 \\
86.5\end{array}$ & $\begin{array}{l}1.5 \\
1.4\end{array}$ & $\begin{array}{l}3.1 \\
0.7\end{array}$ & $\begin{array}{l}81.9 \\
82.8\end{array}$ & $\begin{array}{l}96.4 \\
94.4\end{array}$ & $\begin{array}{l}14.5 \\
11.6\end{array}$ \\
\hline $\begin{array}{l}\text { Federal } \\
\text { 8. Michoacán }\end{array}$ & $\begin{array}{l}85.8 \\
86.8\end{array}$ & $\begin{array}{l}84.7 \\
85.9\end{array}$ & $\begin{array}{l}88.5 \\
87.9\end{array}$ & $\begin{array}{l}1.1 \\
1.9\end{array}$ & $\begin{array}{l}2.7 \\
1.1\end{array}$ & $\begin{array}{l}66.3 \\
77.4\end{array}$ & $\begin{array}{l}94.1 \\
97.3\end{array}$ & $\begin{array}{l}27.8 \\
19.9\end{array}$ \\
\hline \multicolumn{9}{|c|}{ Grupo III } \\
\hline $\begin{array}{l}\text { Entidad } \\
\text { Federativa }\end{array}$ & Mediana & \multicolumn{2}{|c|}{ Bisagras $^{2}$} & \multicolumn{2}{|c|}{ Distancia (M) } & \multicolumn{2}{|c|}{ Valores extremos } & Rango \\
\hline $\begin{array}{l}\text { 1. Colima } \\
\text { 2. Sonora } \\
\text { 3. Tamaulipas } \\
\text { 4. Quintana Roo } \\
\text { 5. México } \\
\text { 6. Campeche } \\
\text { 7. Tlaxcala } \\
\text { 8. Yucatán }\end{array}$ & $\begin{array}{l}72.7 \\
75.0 \\
76.2 \\
77.1 \\
78.2 \\
78.5 \\
78.8 \\
78.9\end{array}$ & $\begin{array}{l}71.3 \\
73.4 \\
74.0 \\
72.7 \\
75.0 \\
77.9 \\
77.9 \\
77.0\end{array}$ & $\begin{array}{l}75.2 \\
77.0 \\
79.6 \\
84.5 \\
81.5 \\
80.0 \\
81.2 \\
81.2\end{array}$ & $\begin{array}{l}1.4 \\
1.6 \\
2.2 \\
4.4 \\
3.2 \\
0.6 \\
0.9 \\
1.9\end{array}$ & $\begin{array}{l}2.5 \\
2.0 \\
3.4 \\
7.4 \\
3.3 \\
1.5 \\
2.4 \\
2.3\end{array}$ & $\begin{array}{l}64.9 \\
71.7 \\
70.4 \\
64.7 \\
70.8 \\
73.4 \\
74.8 \\
73.1\end{array}$ & $\begin{array}{l}90.8 \\
89.3 \\
97.3 \\
96.5 \\
95.0 \\
95.4 \\
98.6 \\
94.0\end{array}$ & $\begin{array}{l}25.9 \\
17.6 \\
26.9 \\
31.8 \\
24.2 \\
22.0 \\
23.8 \\
20.9\end{array}$ \\
\hline
\end{tabular}


Cuadro 5. México: Medidas obtenidas en el análisis exploratorio de datos (mediana, bisagras, distancia, entre mediana y bisagras (M), valores extremos y rango) por grupos de entidades federativas, 195 1-1975

\section{(Series de porcentajes en 24 años) ${ }^{1}$ (continuación)}

\begin{tabular}{|c|c|c|c|c|c|c|c|c|}
\hline \multicolumn{9}{|c|}{ Grupo IV } \\
\hline \multirow{2}{*}{$\begin{array}{l}\text { Entidad } \\
\text { Federativa }\end{array}$} & \multirow[t]{2}{*}{ Mediana } & \multicolumn{2}{|c|}{ Bisagras $^{2}$} & \multicolumn{2}{|c|}{ Distancia (M) } & \multicolumn{2}{|c|}{ Valores extremos } & \multirow[t]{2}{*}{ Rango } \\
\hline & & inferior & Superior & Inferior & Superior & Inferior & Superior & \\
\hline 1. Morelos & 65.6 & 63.4 & 68.5 & 2.2 & 2.9 & 59.7 & 91.9 & 32.2 \\
\hline 2. Puebla & 66.3 & 63.3 & 69.0 & 3.0 & 2.7 & 59.3 & 91.4 & 32.1 \\
\hline 3. Baja Calif. Sur & 69.7 & 67.6 & 76.4 & 2.1 & 6.7 & 61.0 & 95.0 & 34.0 \\
\hline \multicolumn{9}{|c|}{ Grupo V } \\
\hline \multirow{2}{*}{$\begin{array}{l}\text { Entidad } \\
\text { Federativa }\end{array}$} & \multirow[t]{2}{*}{ Mediana } & \multicolumn{2}{|c|}{ Bisagras $^{2}$} & \multicolumn{2}{|c|}{ Distancia (M) } & \multicolumn{2}{|c|}{ Valores extremos } & Rango \\
\hline & & Inferior & Superior & Inferior & Superior & Inferior & Superior & \\
\hline 1. Hidalgo & 52.4 & 50.3 & 57.8 & 2.1 & 5.4 & 42.8 & 90.3 & 47.5 \\
\hline 2. Chiapas & 52.7 & 49.0 & 57.1 & 3.7 & 4.4 & 39.7 & 89.2 & 49.5 \\
\hline 3. Sinaloa & 55.6 & 52.1 & 62.0 & 3.5 & 6.4 & 49.5 & 90.0 & 40.5 \\
\hline 4. Nayarit & 58.3 & 55.8 & 61.2 & 2.5 & 2.9 & 51.5 & 80.6 & 29.1 \\
\hline 5. Tabasco & 58.3 & 55.5 & 65.6 & 2.8 & 7.3 & 47.2 & 92.4 & 45.2 \\
\hline
\end{tabular}


Cuadro 5. México: Medidas obtenidas en el análisis exploratorio de datos (mediana, bisagras, distancia, entre mediana y bisagras (M), valores extremos y rango) por grupo de entidades federativas, 1951-1975

(Series de porcentajes en 24 años) $)^{1}$ (conclusión)

\begin{tabular}{|c|c|c|c|c|c|c|c|c|}
\hline \multicolumn{9}{|c|}{ Grupo VI } \\
\hline \multirow{2}{*}{$\begin{array}{l}\text { Entidad } \\
\text { Federativa }\end{array}$} & \multirow[t]{2}{*}{ Mediana } & \multicolumn{2}{|c|}{ Bisagras $^{2}$} & \multicolumn{2}{|c|}{ Distancia (M) } & \multicolumn{2}{|c|}{ Valores extremos } & \multirow[t]{2}{*}{ Rango } \\
\hline & & Inferior & Superior & Inferior & Superior & Inferior & Superior & \\
\hline 1. Veracruz & 49.7 & 45.0 & 55.5 & 4.7 & 5.8 & 40.2 & 77.7 & 37.5 \\
\hline 2. Oaxaca & 49.6 & 47.7 & 60.3 & 1.9 & 10.7 & 45.8 & 81.0 & 35.2 \\
\hline
\end{tabular}

Fuente: Cálculos elaborados con base en datos de los Anuarios Estadísticos de los Estados Unidos Mexicanos, 1951-1975, Dirección

1 En el cálculo de las medidas se tomaron en cuenta series de porcentajes de 24 años: los años 1951 y los del periodo 1953-1975.

2 Véase nota 2 del cuadro 3 . 
poco menor que las del grupo anterior, pero como en éste, su tendencia ha sido mantener esa proporción constante, con excepción del Distrito Federal que presenta un rango mucho mayor que el de las otras entidades, por lo que analizamos las proporciones y encontramos que sólo para 1970 fue del orden de 60; para todos los demás años la proporción se mantuvo por encima de $80 \%$. Esto se ve reflejado en el valor de $(M)$, que es similar en magnitud a la de las otras entidades que conforman este grupo. $\mathrm{Al}$ igual que en el grupo $\mathrm{I}$ estas entidades aparentemente tienen características económicas similares, con excepción del estado de Guerrero.

\section{Grupo III}

El grupo III se compone también de 8 entidades, pero más diversificadamente, ya que se tienen dos estados del norte (Sonora y Tamaulipas), dos del centro (México y Tlaxcala), tres estados del sureste (Quintana Roo, Campeche y Yucatán) y uno del sur Pacífico (Colima). Para este grupo la mediana está en los setenta (72-79) y la dispersión es un poco mayor que para los grupos anteriores, como se puede ver por las distintas (M) y sobre todo por el rango, que es mayor que para los dos grupos anteriores. La entidad con mayores variaciones es Quintana Roo, que tiene un rango del doble con respecto a los de este grupo y que en el análisis de la serie completa sí se observan variaciones de un año a otro. Las características económicas de este grupo parecen ser bastante diversas, aunque los del sureste podrian formar una unidad debido a la homogeneidad de características socioeconómicas que guardan entre sí.

\section{Grupo IV}

Este grupo está formado sólo por tres entidades (Morelos, Puebla y Baja California Sur). La mediana es del orden de 65 a 70 y su dispersión o variación de la proporción de legítimos es un poco mayor que la del grupo III. En una primera aproximación diriamos que tampoco son entidades con características económicas muy similares. 


\section{Grupo V}

Para este grupo el porcentaje de legítimos sólo alcanza entre el 50 y $60 \%$. Es decir una proporción bastante menor en comparación con las entidades del primer grupo. La dispersión y las variaciones de estas proporciones, son también más notorias que en los grupos anteriores, con una (M) mayor que en los otros grupos y un rango promedio de cerca de 20. Las características económicas en común entre las entidades del Pacífico norte (Nayarit y Sinaloa), las del sureste (Chiapas y Tabasco), y el estado de Hidalgo parecen muy difíciles de establecer.

\section{Grupo VI}

Este grupo está formado sólo por dos entidades: Veracruz y Oaxaca. Se distingue de los anteriores porque éstas son las únicas entidades cuya proporción de ilegítimos rebasa a la de legítimos, ya que la mediana es del orden de 49. Además de lo anterior- estas entidades se distinguen porque su dispersión y sus variaciones son también mayores. El rango es de 20 y la (M) es también un poco mayor que en el grupo anterior. Las caracteristicas de estas entidades hay que estudiarlas con atención, dado que en los indicadores económicos parecen ser bastante diferentes.

Las principales conclusiones que surgen del análisis de la información sobre las proporciones de nacimientos legítimos a nivel de entidad federativa son:

a) El porcentaje de hijos legítimos varía de entidad a entidad. De acuerdo con los indicadores utilizados pudimos identificar seis grupos diferentes.

b) Cada uno de estos grupos no son sólo diferentes en la proporción de hijos legítimos, que varía entre 90 y $49 \%$, sino también en la tendencia que han seguido: dispersión y variaciones.

c) Para ninguna de las entidades se observa una tendencia de descenso o de incremento de la proporción de nacimientos legítimos. La proporción se mantiene casi constante, a través del periodo analizado para la mayoría de las entidades.

d) Las entidades con una proporción menor de nacimientos legítimos presentan una mayor dispersión en las proporciones entre las entidades, y también grandes variaciones de un año a otro.

e) Es difícil encontrar una razón para la agrupación encontrada, pues las entidades que conforman algunos de los grupos tienen características socioeconómicas muy diferentes entre sí. 
f) Es necesario profundizar más en el análisis socioeconómico de las entidades para encontrar una explicación de las diferencias en las proporciones de nacimientos ilegítimos. Esta explicación tal vez se encuentre más en variables de tipo social, como la organización familiar que predomine en cada entidad, que en las de tipo económico.

g) No hay que descartar la posibilidad de que las diferencias encontradas en la información que puede haber de una entidad a otra podrían estar significando una calidad menor en la información.

h) Otro factor importante para analizar la agrupación obtenida es la discrepancia entre el concepto de ilegitimidad definido por la ley y el que utiliza la Dirección General de Estadística. Esta discrepancia puede traer confusión para las oficialías del registro civil y ser la responsable de las diferencias encontradas.

RELACIÓN ENTRE LA CONDICIÓN DE LEGITIMIDAD EN EL REGISTRO DE NACIMIENTOS, EL TIPO DE UNIÓN Y EL REGISTRO TARDIOO

En esta parte del trabajo se intentará encontrar la relación que pudiera existir en las entidades federativas, entre el tipo de unión que prevalece en cada entidad y la proporción de nacimientos según su condición. También a nivel de entidades federativas se procurará ver si existe alguna relación entre la proporción de nacimientos ilegítimos y la proporción de registros tardíos.

De acuerdo con la definición sobre la condición de legitimidad que hemos visto en la sección anterior, la proporción de nacimientos ilegítimos y la de uniones consensuales tendrían una relación directa, puesto que todo nacimiento proveniente de una unión consensual automáticamente queda registrado en las estadísticas como ilegítimo. Lo que queda por definir entonces es la forma de relación entre ambas proporciones.

En el cuadro 6 se presenta la proporción de población unida consensualmente respecto al total de la población unida, según los censos de población de 1950, 1960 y 1970 , para las 32 entidades federativas. Con la misma metodología que utilizamos para estudiar las tendencias de las proporciones de los nacimientos legítimos, se analizó la información sobre uniones consensuales. El resultado de este análisis se resume en el cuadro 7 , del cual podemos decir lo siguiente.

8 Esta sección del trabajo tiene un carácter preliminar. 
Cuadro 6. Porcentajes de la población unida consensualmente respecto al total de la población unida por entidad federativa: 1950,1960 y 1970

\begin{tabular}{lrrr}
\hline Entidad & $1950^{1}$ & $1960^{2}$ & $1970^{2}$ \\
\hline Federativa & 17.66 & 14.10 & 13.72 \\
\hline Total del País & & & 3.69 \\
1. Aguascalientes & 5.45 & 4.24 & 12.54 \\
2. Baja California Norte & 15.17 & 14.07 & 11.43 \\
3. Baja California Sur & 16.82 & 13.17 & 12.51 \\
4. Campeche & 20.31 & 13.21 & 7.24 \\
5. Coahuila & 10.39 & 8.30 & 11.84 \\
6. Colima & 15.09 & 11.12 & 33.80 \\
7. Chiapas & 47.41 & 40.40 & 10.79 \\
8. Chihuahua & 12.25 & 9.95 & 8.08 \\
9. Distrito Federal & 15.52 & 11.02 & 11.68 \\
10. Durango & 14.54 & 10.40 & 3.52 \\
11. Guanajuato & 3.80 & 3.09 & 13.41 \\
12. Guerrero & 15.41 & 11.42 & 24.05 \\
13. Hidalgo & 38.13 & 27.27 & 5.34 \\
14. Jalisco & 6.11 & 4.73 & 8.90 \\
15. México & 9.83 & 8.03 \\
16. Michoacán & 5.98 & 4.81 & 6.38 \\
17. Morelos & 25.94 & 19.36 & 20.16 \\
18. Nayarit & 28.72 & 23.17 \\
19. Nuevo León & 7.27 & 5.95 & 5.97
\end{tabular}


20. Oaxaca

21. Puebla

22. Querétaro

23. Quintana Roo

24. San Luis Potos

25. Sinaloa

6. Sonora

27. Tabasco

28. Tamaulipas

29. Tlaxcala

30. Veracruz

31. Yucatán

32. Zacatecas

21.70
18.45
4.80
27.97
14.64
39.54
19.21
37.87
16.27
12.25
35.37
19.42
5.44

11.21

31.21

16.50
30.26

30.26
14.36

14.36
10.76

30.87

1.86

Fuente. Censos de población 1950, 1960 y 1970, Dirección General de Estadistica.

En el censo de 1950 la pregunta sobre estado civil se hizo a las mujeres de 14 años y más, y a los hombres de 16 años y más.

2 En los censos de 1960 y 1970 la pregunta sobre estado civil se hizo a la población de 12 años y más, tanto para hombres como para mujeres. 
Cuadro 7. Resumen de las medidas obtenidas en el análisis exploratorio de los porcentajes de población unida consensualmente: 1950,1960 y 1970

\begin{tabular}{lcrcccccc}
\hline Año & \multirow{2}{*}{ Mediana } & \multicolumn{2}{c}{ Bisagras } & \multicolumn{2}{c}{ Distancia (M) } & \multicolumn{2}{c}{ Valores extremos } & Rango \\
& & Inferior & Superior & Inferior & Superior & Inferior & Superior & \\
\hline 1950 & 15.5 & 10.1 & 23.8 & 5.4 & 8.3 & 3.8 & 47.4 & 43.6 \\
1960 & 11.7 & 8.2 & 19.2 & 3.5 & 7.5 & 3.1 & 40.4 & 37.3 \\
1970 & 11.9 & 7.7 & 17.8 & 4.2 & 5.9 & 3.5 & 33.8 & 30.3 \\
\hline
\end{tabular}

Fuente: Calculos elaborados con base en los datos del cuadro 6. 
La proporción de uniones consensuales ha disminuido con el tiempo. El cambio mayor se da de 1950 a 1960 , ya que la mediana en 1950 era de 15.5 y en 1960 y 1970 el valor de ésta fue de 11.7 y 11.9, respectivamente. Las bisagras nos indican que la distribución es asimétrica hacia los valores superiores. Las diferencias entre las entidades federativas también disminuyen a través del tiempo como to demuestra el valor de (M) y del rango que de 43.0 en 1950 desciende hasta 30.3 en 1970.

Ya en este primer análisis encontramos diferencias en el comportamiento entre las proporciones de uniones consensuales y nacimientos ilegítimos. Como estos últimos son un complemento de la proporción de nacimientos legítimos que analizamos en el apartado anterior, sabemos que no hubo una tendencia de descenso y que las diferencias entre entidades federativas se mantuvieron a lo largo del periodo considerado.

En la gráfica 3.A elaborada con datos de los cuadros 6 y 8 se presentan los datos de la proporción de uniones consensuales para 1950 y la proporción de nacimientos ilegítimos de 1951, para las 32 entidades federativas.

En el eje $(X)$ hicimos una gráfica de la proporción de uniones consensuales y en el eje $(Y)$ de la proporción de nacimientos ilegítimos. Seguimos este mismo procedimiento para los datos de 1960 y 1970, descrito en las gráficas 3.B y 3.C.

En la recta que suponemos que indica esta relación, consideramos a la proporción de uniones consensuales como la variable independiente $(X)$, y a la proporción de nacimientos ilegítimos como la variable dependiente $(Y)$. Las rectas de regresión que resultaron se estiman como:

$$
\begin{gathered}
Y_{1950=}=3.66453+1.32545 X_{1950} \\
t=12.47 ; R^{2}=0.8382 \\
Y_{1960=}=5.09856+1.44017 X_{1960} \\
t=11.5 ; R^{2}=0.8151 \\
Y_{1970=}=8.02343+1.21304 X_{1970} \\
t=8.3 ; R^{2}=0.6983
\end{gathered}
$$

Lo primero que destaca al comparar estas tres ecuaciones es que la pendiente es mayor que uno para los tres casos. Otro punto importante es que los coeficientes de correlación para 1950 y 1960 
Cuadro 8. México: porcentajes de registro de nacimientos ilegítimos al total de nacimientos registrados, por entidad federativa, 1951, 1960 y 1970

\begin{tabular}{|c|c|c|c|}
\hline $\begin{array}{l}\text { Entidad } \\
\text { Federativa }\end{array}$ & 1951 & 1960 & 1970 \\
\hline Total del País & 27.3 & 25.0 & 27.4 \\
\hline 1. Aguascalientes & 8.6 & 9.4 & 11.2 \\
\hline 2. Baja California Norte & 21.9 & 20.5 & 14.1 \\
\hline 3. Baja California Sur & 39.0 & 32.4 & 23.6 \\
\hline 4. Campeche & 21.3 & 21.7 & 21.7 \\
\hline 5. Coahuila & 10.9 & 8.8 & 14.4 \\
\hline 6. Colima & 19.1 & 27.9 & 24.8 \\
\hline 7. Chiapas & 60.3 & 52.6 & 45.2 \\
\hline 8. Chihuahua & 13.8 & 15.3 & 11.2 \\
\hline 9. Distrito Federal & 16.9 & 14.2 & 33.7 \\
\hline 10. Durango & 23.6 & 19.1 & 22.6 \\
\hline 11. Guanajuato & 17.3 & 15.7 & 13.4 \\
\hline 12. Guerrero & 23.4 & 19.2 & 23.4 \\
\hline 13. Hidalgo & 57.2 & 50.8 & 42.2 \\
\hline 14. Jalisco & 11.5 & 9.7 & 17.0 \\
\hline 15. México & 27.2 & 25.3 & 29.2 \\
\hline 16. Michoacán & 12.7 & 12.8 & 13.9 \\
\hline 17. Morelos & 37.5 & 36.1 & 29.8 \\
\hline 18. Nayarit & 48.5 & 45.1 & 40.8 \\
\hline 19. Nuevo León & 6.1 & 5.7 & 6.8 \\
\hline 20. Oaxaca & 49.3 & 50.4 & 47.8 \\
\hline 21. Puebla & 37.9 & 36.7 & 39.1 \\
\hline 22. Querétaro & 7.6 & 8.9 & 9.9 \\
\hline 23. Quintana Roo & 35.7 & 27.6 & 15.6 \\
\hline 24. San Luis Potosí & 20.1 & 16.0 & 18.5 \\
\hline 25. Sinaloa & 50.5 & 46.7 & 37.4 \\
\hline 26. Sonora & 20.6 & 26.4 & 24.9 \\
\hline 27. Tabasco & 52.8 & 45.0 & 37.7 \\
\hline 28. Tamaulipas & 18.1 & 23.7 & 26.9 \\
\hline 29. Tlaxcala & 24.4 & 21.5 & 25.3 \\
\hline 30. Veracruz & 59.8 & 56.0 & 48.9 \\
\hline 31. Yucatán & 26.9 & 23.0 & 21.0 \\
\hline 32. Zacatecas & 9.2 & 8.2 & 7.1 \\
\hline
\end{tabular}

Fuente: Anuarios Estadísticos de los Estados Unidos Méxicanos, 1951, 1960 y 1970, Dirección General de Estadística. 


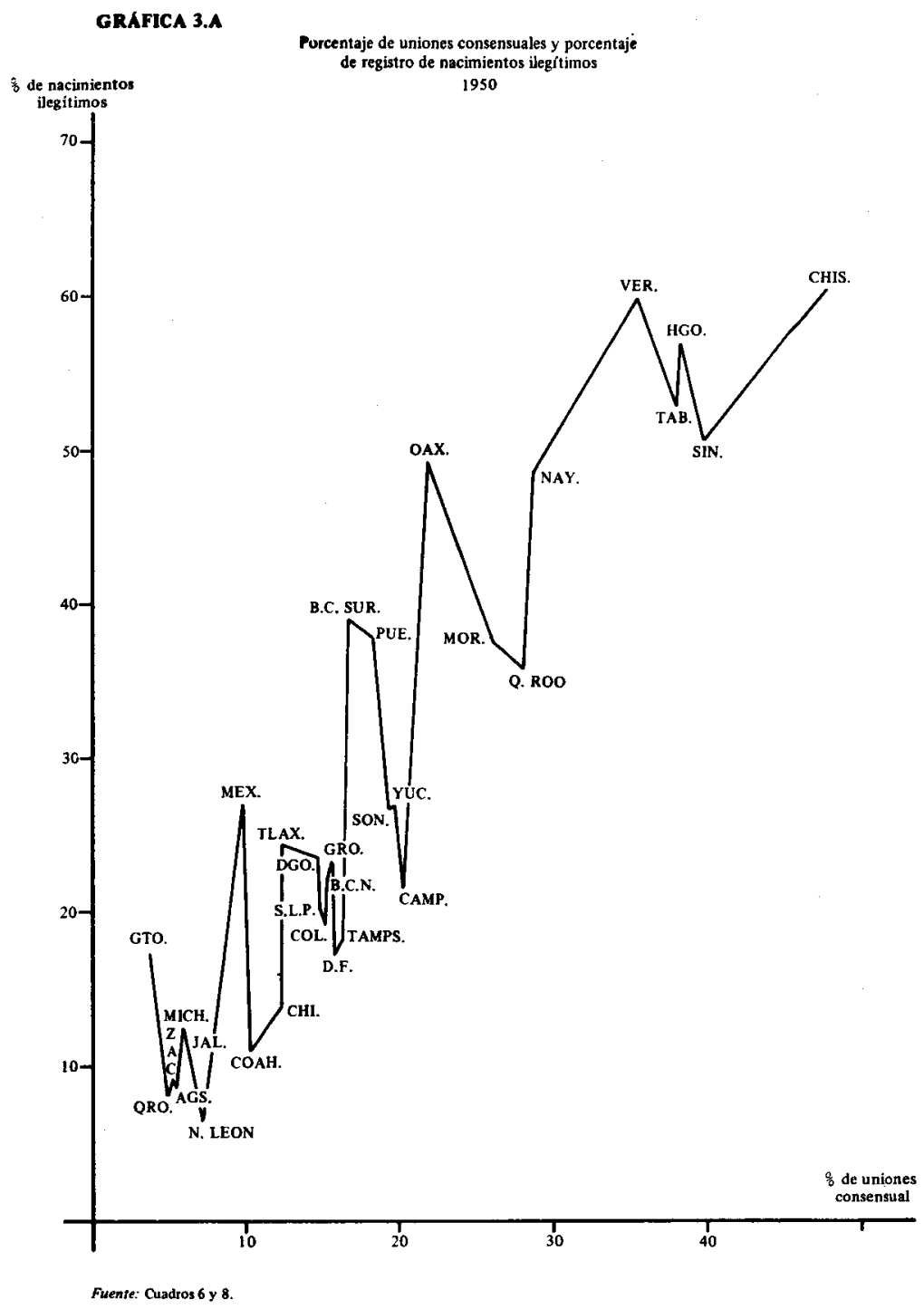




\section{GRAFICA 3.8}

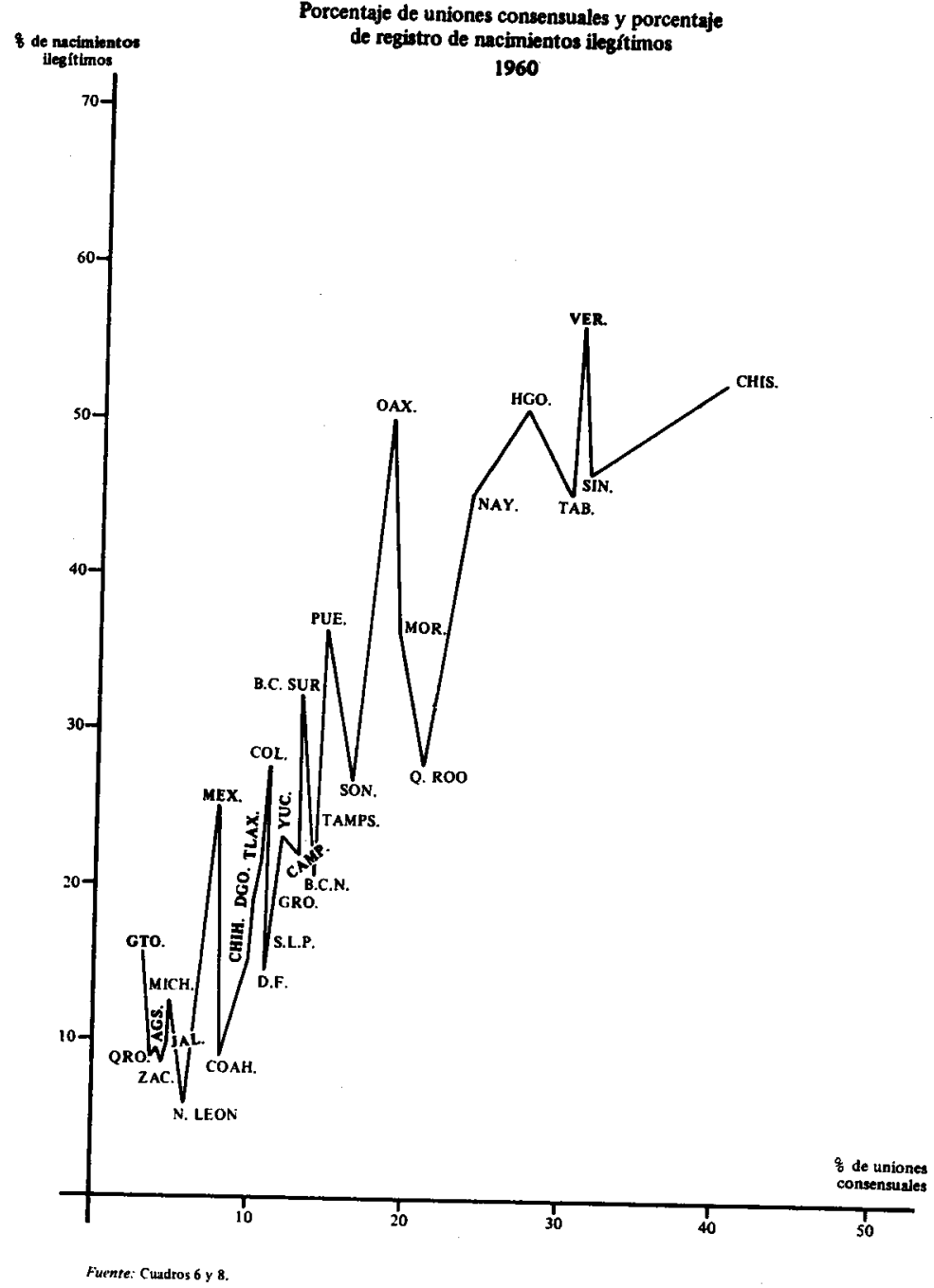




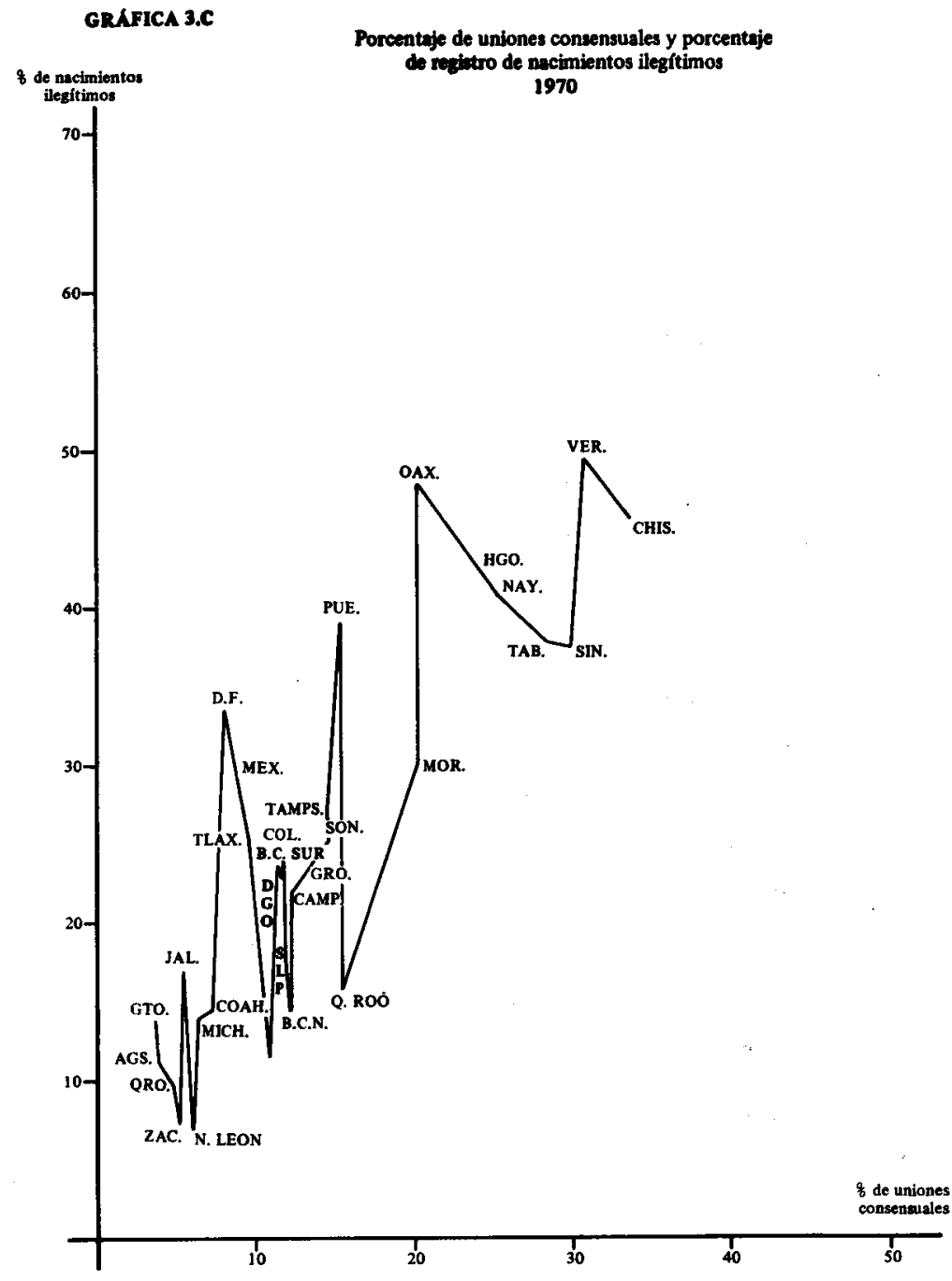

Fuente: Cuadros 6 y 8 
son bastante altos, no así para 1970 en que resulta 0.7 , aunque este último no es bajo tampoco.

Estos datos, aunque elementales, señalan una relación estadística de tipo lineal entre las dos variables. Queda por explicar la naturaleza y la causa de esta relación.

Otra variable relacionada con la unión consensual y los nacimientos ilegítimos parece ser el registro tardío de los nacimientos (véase cuadro 9). En vista de los resultados anteriores que encuentran una relación lineal entre la proporción de uniones consensuales y la de nacimientos ilegítimos, es lícito pensar en una relación parecida entre la proporción de nacimientos ilegítimos y la de nacimientos tardiamente registrados. En las gráficas 4.A, 4.B, y 4.C se presentan los datos correspondientes a estas variables para los años 1950, 1960 y 1970.

A continuación se consignan los resultados de las regresiones lineales propuestas:

$$
\begin{aligned}
Y_{50} & =5.4628-0.00705 X_{50} \\
t & =-0.149 ; R^{2}=0.007 \\
Y_{60} & =4.33678+0.10527 X_{60} \\
t & =1.967 ; R^{2}=0.1142 \\
Y_{70} & =8.00121+0.23922 X_{60} \\
t & =2.207 ; R^{2}=0.1397
\end{aligned}
$$

Como puede observarse a través de los resultados de las regresiones en têrminos de los parámetros estimados y de los coeficientes de correlación, no se puede garantizar que exista un modelo lineal que describa la interdependencia.

Las conclusiones preliminares de esta sección son:

a) Sí es posible establecer una relación lineal entre la proporción de uniones consensuales de una entidad y la proporción de hijos ilegítimos. Falta explicar el porqué de este tipo de relación y de los cambios en la pendiente de las rectas encontradas.

b) No hay una relación lineal entre la proporción de hijos ilegítimos y la proporción de registro tardío. 
Cuadro 9. México: Porcentajes de personas registradas con un año y más de edad respecto al total de registros, por entidad federativa: 1950,1960 y 1970

\begin{tabular}{|c|c|c|c|}
\hline $\begin{array}{l}\text { Entidad } \\
\text { Federativa }\end{array}$ & 1950 & 1960 & 1970 \\
\hline \multicolumn{4}{|l|}{ Total del $\mathrm{Pa}$ is } \\
\hline 1. Aguascalientes & 1.9 & 2.0 & 5.9 \\
\hline 2. Baja California Norte & 5.2 & 8.7 & 11.5 \\
\hline 3. Baja California Sur & 4.6 & 8.3 & 15.5 \\
\hline 4. Campeche & 4.8 & 3.8 & 8.4 \\
\hline 5. Coahuila & 5.1 & 6.4 & 11.6 \\
\hline 6. Colima & 3.1 & 2.4 & 8.7 \\
\hline 7. Chiapas & 4.5 & 7.5 & 11.9 \\
\hline 8. Chihuahua & 4.0 & 4.7 & 8.5 \\
\hline 9. Distrito Federal & 17.5 & 14.5 & 21.4 \\
\hline 10. Durango & 7.2 & 6.2 & 8.9 \\
\hline 11. Guanajuato & 3.0 & 5.0 & 17.9 \\
\hline 12. Guerrero & 5.1 & 8.9 & 19.5 \\
\hline 13. Hidalgo & 3.8 & 8.9 & 16.1 \\
\hline 14. Jalisco & 2.6 & 4.3 & 7.3 \\
\hline 15. México & 3.8 & 7.7 & 17.8 \\
\hline 16. Michoacán & 1.1 & 4.5 & 12.4 \\
\hline 17. Morelos & 6.9 & 6.2 & 16.4 \\
\hline 18. Nayarit & 2.3 & 4.1 & 8.2 \\
\hline 19. Nuevo León & 11.5 & 8.7 & 11.7 \\
\hline 20. Oaxaca & 0.5 & 1.1 & 8.7 \\
\hline 21. Puebla & 3.0 & 7.5 & 16.1 \\
\hline 22. Querétaro & 3.5 & 3.4 & 8.2 \\
\hline 23. Quintana Roo & 3.7 & 5.8 & 35.4 \\
\hline 24. San Luis Potosí & 4.4 & 7.0 & 8.5 \\
\hline 25. Sinaloa & 5.8 & 13.2 & 21.7 \\
\hline 26. Sonora & 6.9 & 7.2 & 10.5 \\
\hline 27. Tabasco & 9.2 & 11.7 & 23.6 \\
\hline 28. Tamaulipas & 19.5 & 19.7 & 25.7 \\
\hline 29. Tlaxcala & 2.4 & 3.8 & 9.0 \\
\hline 30. Veracruz & 9.3 & 20.0 & 34.1 \\
\hline 31. Yucatán & 1.4 & 2.1 & 3.6 \\
\hline 32. Zacatecas & 0.9 & 1.1 & 3.4 \\
\hline
\end{tabular}

Fuente: Anuarios Estadísticos de los Estados Unidos Mexicanos, 1950, 1960 y 1970 , Dirección General de Estadística. 


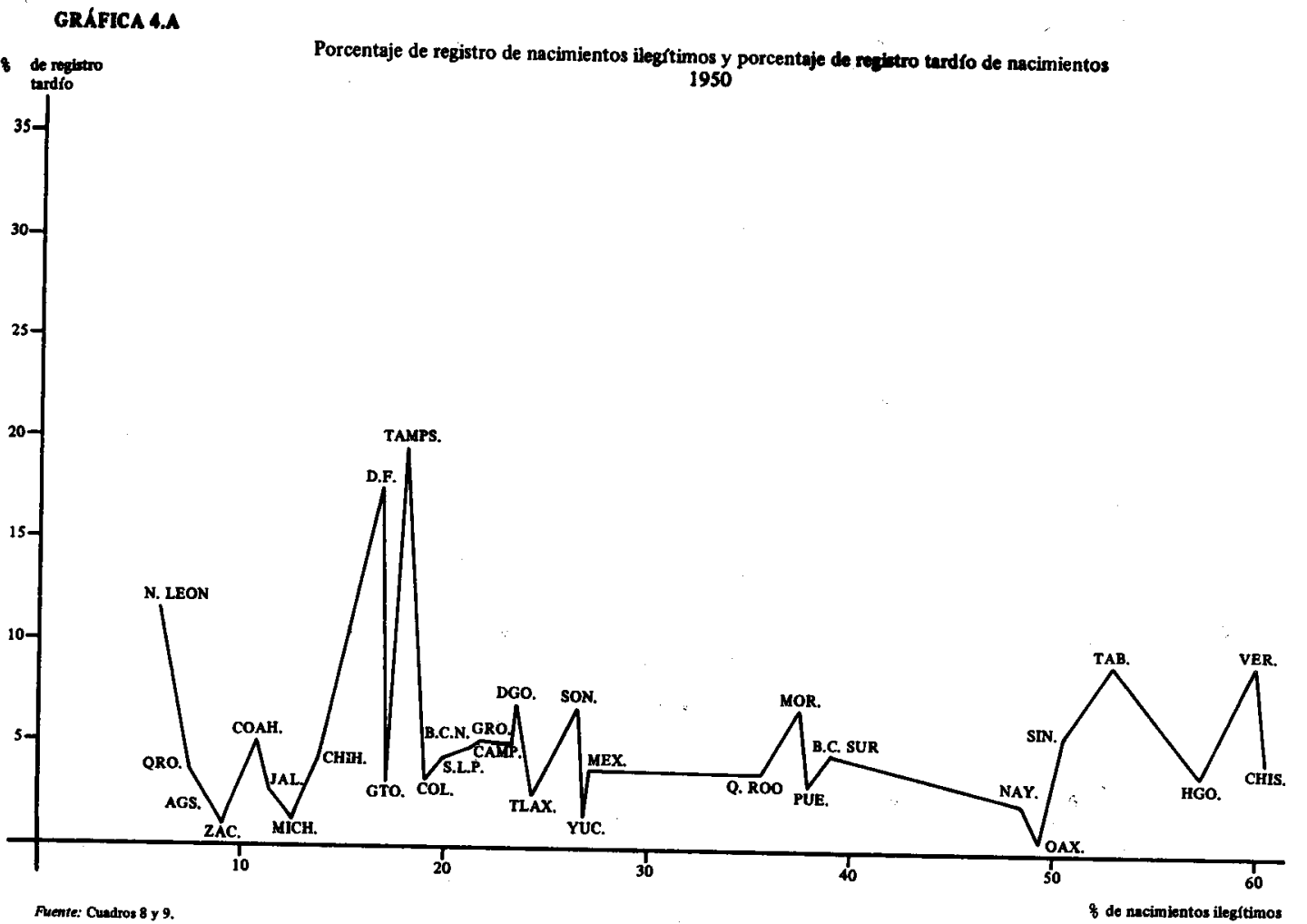




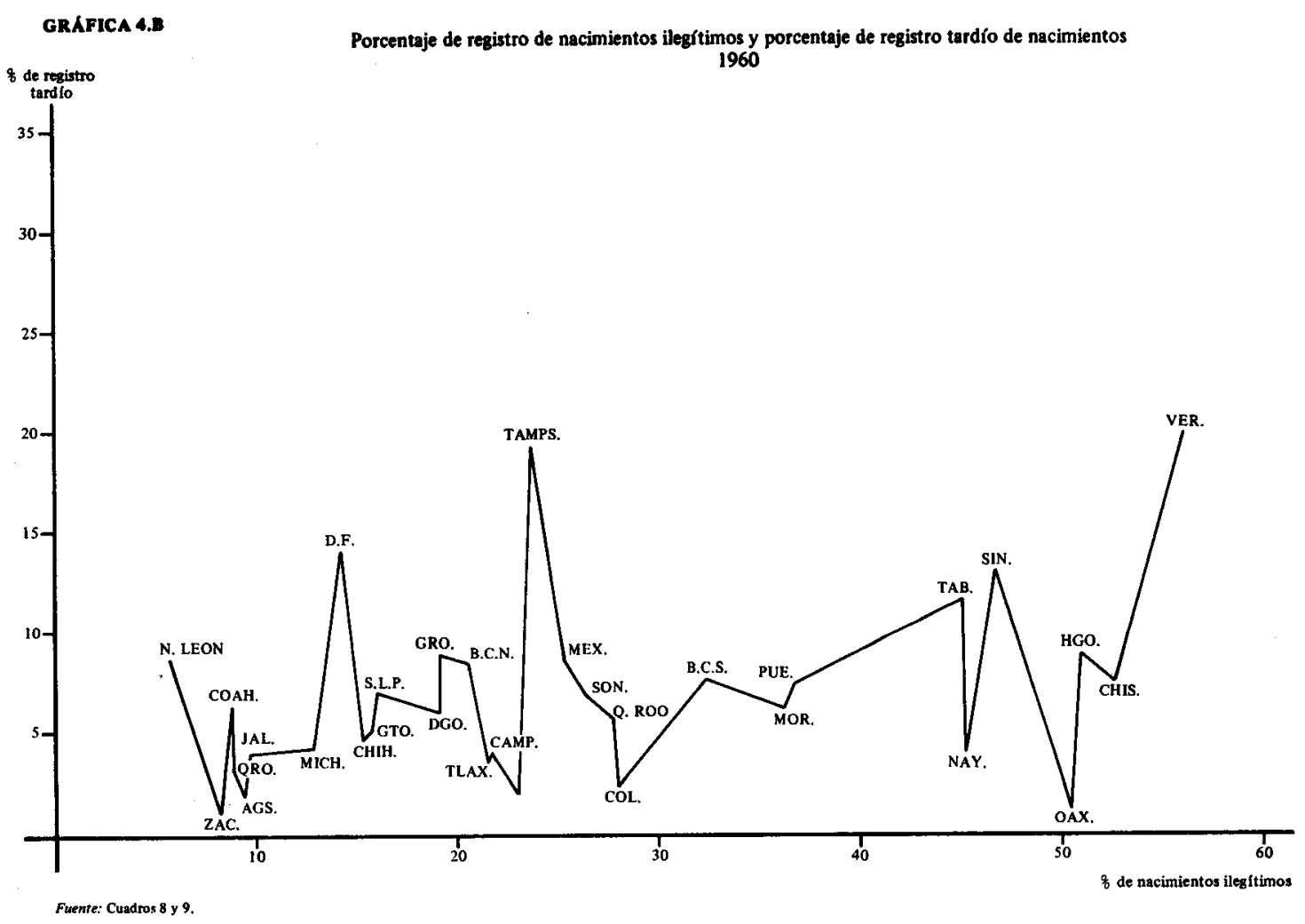




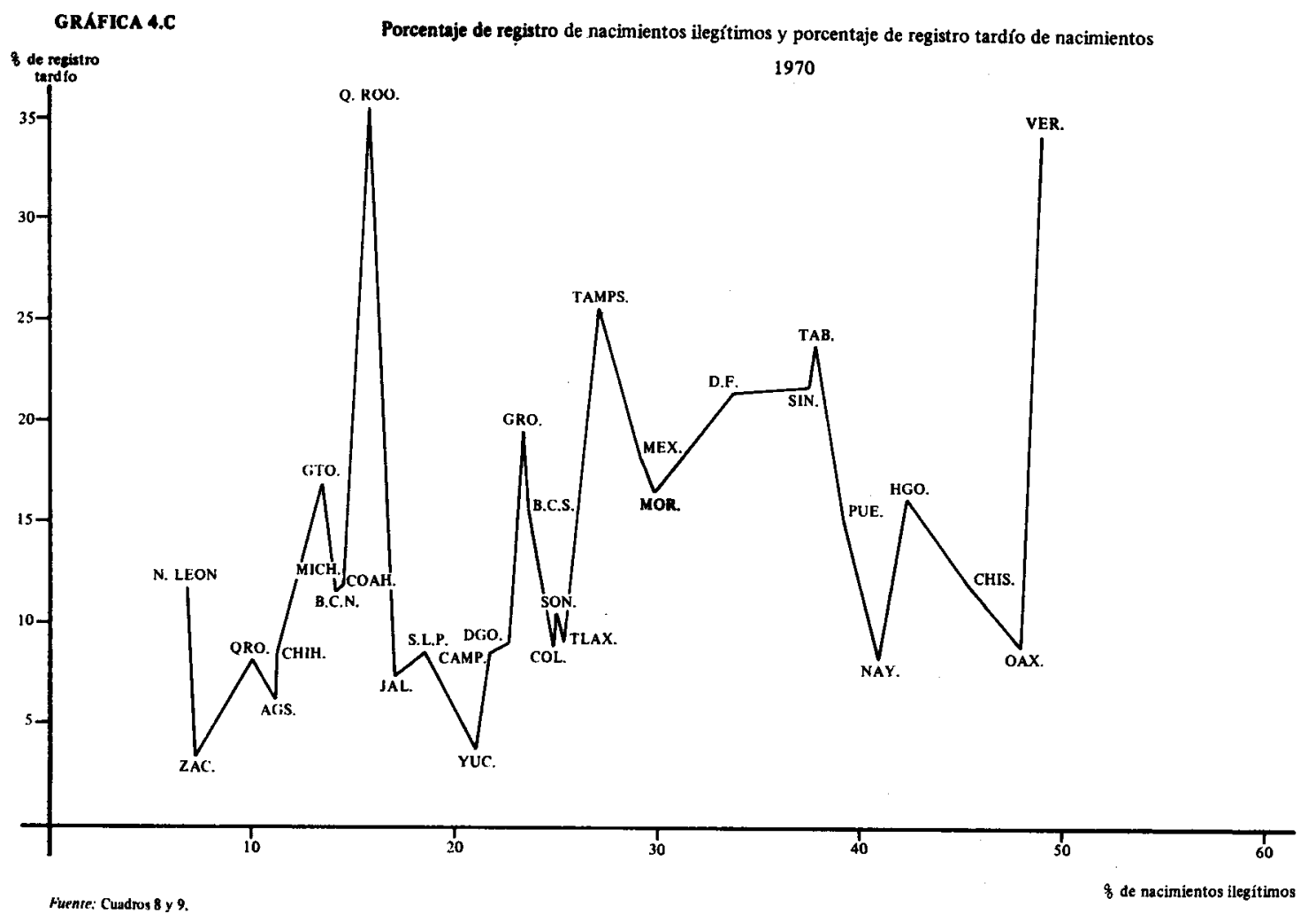




\section{Bibliografía}

DiRECCIÓN GENERAL DE EsTAdístICA, Informe sobre las principales estadisticas, Secretaría de la Economía Nacional, 1941, 174 pp.

- Normas para las publicaciones del área de estadisticas demográficas y sociales, programas y procedimientos, serie II, núm. 2, Secretaría de Programación y Presupuesto, 1975.

- Instrucciones para llenar en el Registro Civil los formularios estadísticos de los hechos vitales, programas y procedimientos, serie II, núm. 10, Secretaría de Programación y Presupuesto, 1975.

Gleason Galicịa, Rubén, Las estadísticas y censos de México, su organización y estado actual, Instituto de Investigaciones Sociales, UNAM, 1968.

QUILODRÁN, Julieta, Impacto de la disolución de uniones sobre la fecundidad en México, 1980 (mimeo).

"Análisis de la nupcialidad a través de la historia de uniones", Investigación demográfica en México, Consejo Nacional de Ciencia y Tecnología, 1978, pp. 139-149.

Secretaría de Gobernación, El Registro Civil en México. Antecedentes histórico-legislativos, aspectos juridicos y doctrinarios, Dirección General del Registro Nacional de Población e Identificación Personal, 1982. 